\title{
Ueber die Alkaloide von Corydalis cava.
}

\author{
Von Dr. H. Zi $\log \in \mathrm{n} b$ ein. \\ (Eingegangen, den 1. VII. 1896.)
}

Die Alkaloide der einzelnen Corydalisarten sind im Laufe der Jahre bereits wiederholt der Gegenstand von Untersuchungen gewesen. Es sei nur an die älteren Arbeiten von Peschier, Winkler, Doebereiner, Ruikholdt, Müller, Leube und Wicke erinnert. In neuerer Zeit haben sich mit der Ermittelung der Zusammensetzung und der Eigenschaften dieser Basen besonders Adermann, 1) Birsmann, 2) Dobbie und Lauder,$\left.^{3}\right)$ Freund und Josephi ${ }^{4}$ ) beschäftigt.

Jedoch hatte schon vor 8 Jahren auf Veranlassung des Herrn Prof. Dr. E. S chmidt,,$^{5}$ Herr $\mathrm{N}$ o elle die Alkaloide der Corydalis cava im hiesigen pharm. chem. Institut näher untersucht.

Das Ergebnis dieser Arbeiten konnte leider nicht veröffentlicht werden, da Herr $\mathrm{Noelle}$ kurz vor Abschlufs derselben durch den Tod abgerufen wurde. Schon damals beobachtete E. S c h mi d t ${ }^{\mathbf{B}}$ ) bei der zwischen $134^{0}-135^{\circ}$ schmelzenden, von $\mathrm{No}$ oll e als Hauptbestandteil in den Corydalisknollen gefundenen, jetzt allgemein mit dem Namen Corydalin belegten Base, dafs dieselbe in ihrem Verhalten am Licht und gegen Jod eine solche Aehnlichkeit mit dem Hydroberberin und dem Canadin zeigte, dals sich die Vermutung aufdrängte, es beständen zwischen den Corydalis- und Berberisalkaloiden direkte chemische Beziehungen.

Auf Veranlassung meines hochverehrten Herrn Lebrers, des Herrn Geh. Rat Prot. Dr. E. S c h mi d t habe ich jene Beziehungen, in denen anscheinend das Corydalin und vielleicht auch ein Teil der übrigen aus den Knollen von Corydalis cava isolierten Basen

1) Inaug. Dissert. Dorpat. 1890 ,

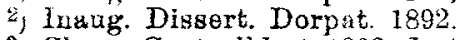

3) Chem. Centralblatt 1892. 1. 442. $632 ; 1892$. II. $220.481 ; 1893$. I. 784 ; Lond. Chem. Soc. 1893 ; Chem. Ztg. 1894.

4) Chem. Centralblatt 1892. II. 418; 1893. II. 923; Annal. d. Chem. 277.

5) Archiv d. Pharm. 1894, p. 143.

6) Archiv d. Pharm. 1894, p. 143. 
zum Berberin stehen, zum Gegenstande weiterer Untersuchungen gemacht. Hierbei habe ich mich bemüht, die zum Toil noch lücken haften und zum Teil einander widersprechenden Angaben über Darstellung, Zusammensetzung und Eigenschaften der einzelnen Basen nach Möglichkeit zu ergänzen.

Meine erste Aufgabe war, die einzelnen Alkaloide in genügender Menge und Reinheit darzustellen. In dankenswerter Weise überliels mir Herr Geh. Rat S ch mi d t die in seinem Besitz befindlichen, aus dem Nachlasse No elle's stammenden reinen und rohen Alkaloide; es waren dies:

1. ein allmählich etwas gelblich gefärbter, bei $134^{0}-135^{0}$ schmelzender, krystallisierter Teil.

2. eine zwischen $120-180^{\circ}$ schmelzende Fraktion, ein gelbliches Pulver darstellend.

3. Gelbe Krystalle, deren Schmelzpunkt bei $217-218^{\circ}$ lag.

4. als Rohalkaloide bezeichnete, pulvrige, graubraune Massen.

5. dunkelbraunes Harz.

a) Verarbeitung der Noelle'schen Alkaloide.

Die bei $134^{\circ}$ und zwischen $120^{\circ}$ und $180^{\circ}$ schmelzenden, nur schwach gelb gefärbten Anteile wurden zur Reinigung zusammen behandelt, indem ich sie in lauwarmem Wasser suspendierte und Salzsäure bis zur schwach sauren Reaktior und damit eintretenden Lösung zusetzte. Es resultierte eine schwach gelb gefärbte Flüssigkeit, die, nach dem Erkalten, in fünfprozentige Natronlauge unter Umrühren eingegossen wurde. Das sich voluminös auscheidende weilse Pulver liels ich kurze Zeit absetzen, saugte die überstehende Flüssigkeit ab, wusch den Niederschlag wiederholt aus, prelste und trocknete ihu bei gewöhnlicher Temperatur. Das rein weils erscheinende Pulver löste ich durch Erwärmen in 96 Proz. Alkohol, aus welchem sich nach dem Erkalten die erste Krystallisation in farblosen Prismen ausschied. Durch Verdunsten des Alkohols bei mäfsiger Wärme errielte ich weitere Krystallisationen, die jedoch mehr oder minder gelb gefürbt waren. Zu ihrer nochmaligen Reinigung löste ich sie wieder in schwacher Salzeäure, fällte diese Lösung durch verdünnte Natronlauge und krystallisierte den Niederschlag aus Alkoholum.

Ich erhielt so farblose Krystalle von Corydalin, welche bei $134^{\circ}$ schmolzen.

In die alkalischen Laugen leitete ich $\mathrm{CO}_{2}$ ein, da ich nach dem zwischen $120^{\circ}$ und $180^{\circ}$ liegenden Schmelzpunkt des angewandten Materials annehmen mufste, dafs darin das in Aetzalkalien lösliche, 
durch kohlensaure Alkalien fällbare, bei 1990 schmelzende andere Hauptalkaloid der Corydalisknollen, das Bulbocapnin, gelöst wäre. Aber erst nach langem Einleiten, als die Flüssigkeit vollständig mit $\mathrm{CO}_{2}$ gesättigt war, trübte sie sich, und es setzte sich allmählich ein geringer Niederschlag zu Boden, den ich abeangte, auswusch und trocknete. In heifsem Alkohol war diese Base sehr schwer löslich. Sie konnte daraus in Gestalt eines weilsen, fein krystallinischen Pulvera erhalten werden vom Schmelzpankt $235^{\circ}$.

Ieh versuchte noch, aus den mit $\mathrm{CO}_{2}$ gesättigten Laugen durch Ausschütteln mit Chloroform Basen zu gewinnen, jedoch war diese Bemühung resultatios.

Das bei $217^{0}-218^{0}$ schmelzende, etwas gelblich gefärbte Alkaloid reinigte ich durch einfaches Umkrystallisieren aus starkem Alkohol und erhielt hierbei direkt weifse, glänzende Tafeln vom Schmelzpunkt $216^{0}-217^{\circ}$.

Die Rohalkaloide und das braune Harz verarbeitete ich gemeinsam. Ich zerrieb die Massen zu einem feinen Pulver und löste dieses nach Möglichkeit in verdinnter, warmer Salzsäure. Die filtrierte, grínlich gefärbte Lösung fällte ich wieder durch verdünnte Natronlauge; jedoch war das ausfallende Pulver stark rotbraun gefärbt, weshalb ich gezwungen war, dasselbe nochmals in Salzsäure zu lösen und diese Lösung von neuem durch Natronlauge zu füllen. Diese Operationen wurden so oft wiederholt, bis ein nur noch grauweils gefärbtes Pulver resultierte. Aus Alkobol krystallisierte die Base auch noch etwas gefärbt, zeigte jedoch den richtigen Schmelzpunkt des Corydalins $134,5^{0}$, weshalb ich sie in dieser Form verwendete. Durch Einleiten von $\mathrm{CO}_{2}$ in die braunen, alkalischen Laugen konnte ich auch hier keine Abscheidung erzielen, ebensowenig wie es mir bis jetzt auf andere Weise gelungen ist, aus diesen Noelle'schen Resten irgend eine der wuiteren Basen zu isolieren.

b. Darstellung der Alkaloide aus Corydalis cava.

Zur Darstellung der Corydalisalkaloide benutzte ich das von Freund und $J$ ose $\mathrm{p} h \mathrm{i}$ angegebene Verfahren von Dr. A l exander Ehrenber ${ }^{1}$ ). $20 \mathrm{~kg}$, von $R \mathrm{um}$ und Lehners- Hannover bezogener Knollen von Corydalis cava wurden grob zerstolsen and im Extraktionsapparat mit 96 Proz, Alkohol circa 14 Tage lang ausgezogen, der Alkohol abdestilliert, der syrupöse Rückstand durch ein Heilswasserfilter filtriert, mit Ammoniak schwach alkalisch gemacht und mit Aether zweimal ausgeschättelt. Die ätherische, grinlich gefärbte Lösung wurde zunächst angefähr auf die Hälfte durch Ab-

1) Annal. d. Chemie 277. p. 4. 
destillieren eingeengt und der Krystallisation überlaseen. Auf diese Weise wurden gewonnen:

I. Grinliche Nadeln, Schmelzpunkt $199-200^{0} 17,0 \mathrm{~g}$.

II. Die weiter eingeengte Mutterlauge lieferte einen fast weifseu Krystallbrei vom Schmelzpunkt $134-150^{\circ} 27,5 \mathrm{~g}$.

III. Von der Lauge wurde noch mehr Aether abdestilliert und ungefähr die doppelte Menge Alkohol zugefügt. Es resultierte nach einiger Zeit eine rein weifse k r s ta 1 l in is c h e Ma s s e vom Schmelzpunkt $133-134^{0}, 20,4 \mathrm{~g}$.

IV. Das syrupartige Extrakt schüttelte ich noch zweimal mit Aether aus, vereinigte die Lösungen, destillierte die Hälfte des Aethers ab und erhielt einen grünlichen Krystallbrei Schmelzpunkt $196^{\circ}, 3,9 \mathrm{~g}$.

$\nabla$. Die Mutterlauge von IV engte ich wieder ein, fügte Alkohol zu und gewann so fast weifse Gebilde vom Schmelzpunkt $182^{\circ}, 4,5 \mathrm{~g}$.

VI. Das ureprüngliche Extrakt schättelte ich hierauf zum fünften und sechsten Male mit Aether aus und erhielt nach dem Abdestilli eren des Aethers fast weifse Krystalle vom Schmelzpunkt 1840, 8,0 g.

VII. Auch die Mutterlaugen von VI. ergaben, eingeengt und mit Alkohol versetzt, grau-weifse Krystalle vom Schmelzpunkt $174^{0}, 1,3 \mathrm{~g}$

Die von III, V und VII übrig gebliebenen alkoholisch-äth erischen Mutterlaugen wurden vereinigt und successive weiter eingeengt. Auf diese Weise wurden gewonnen:

VIII. Weiche, weifse Nadeln Schmelzpunkt $198^{\circ}, 3,9 \mathrm{~g}$.

IX. Kleinkrystallinisches, gelbliches Pulver vom Schmelzpunkt $134^{0}-160^{0}, 1.6 \mathrm{~g}$.

X. Farblos $甘$ Säulon $134^{0}-135^{0}, 1,4 \mathrm{~g}$.

XI. Desgleichen $134^{0}-135^{0}, 2,9 \mathrm{~g}$.

Da nach weiterem Verdunsten keine Krystallabscheidung mehr eintrat, wurde die braungefärbte, dickflïssige. Lösung mit Salzsäure angesäuert, durch Erwärmen die Reste von Alkohol und Aether verjagt, die grün gefärbte Flüssigkeit mit Wasser stark verdünnt und mit Natronlauge schwach alkalisch gemacht. Der entstandene graue Niederschlag wurde abgesaugt, ausgewaschen und mit Aether geschüttelt.

XII. Die erhaltene ätherische Lösung wurde hierauf ein geengt und der Krystallisation überlassen; so wurden erhalten gelbliche, pulverförmige Massen. Schmelzpunkt 1830, 13,0 g.

XIII. Nach weiterem Abdestillieren resultierten noch weilse Krystalle vom Schmelzpunkt $134-135^{\circ}, 4,8 \mathrm{~g}$.

Die von XIII restierende ätherische Lauge wurde fast zur Trockne eingedampft, verdünnte Bromwaseerstoffsäure zugefügt, die Lösung filtriert und zur Krystallisation bei Seite gestellt. Es gelang aber nicht, gui ausgebildete Krystalle zu erhalten, auch ergaben die durch Natronlauge und Natriumkarbonat erhaltene Füllung nach dem Auflösen in Alkohol keine Krystalle. 
Das noch vorbandene ursprüngliche Extrakt verarbeitete ich weiter, indem ich es mit Salzsäure ansäuerte, den Aother durch Eindampfen entfernte, die Lösung mit Natriumkarbonat alkalisch machte und wiederholt mit Chloroform ausschüttelte.

Nach dem Abdestillieren des Chloroforms löste ich den Rückstand in Salzsäure, versetzte die Lösung mit Natriumkarbonat, löste den entstandenen Niederschlag wieder, füllte von Nexem und wiederholte diese Operation noch so oft, bis der Niederschlag nur noch grau gofärbt erschien. Diesen verarbeitete ich dann gemeinsam mit auderen woiter.

Nach dem angegebenen Verfahren hatte ich erhalten $110,2 \mathrm{~g}$ Rohalkaloide, aufserdom die durch die Chloroformausschättelung gewonnene Menge. Die Reinigung der einzelnen Fraktionen richtete sich nach dem Schmelzpunkt derselben.

Fraktion I und VIII wurden aus Alkohol umkrystallisiert und ergaben B ulb o cap $\mathbf{n}$ in vom Schmelzpunkt $199^{\circ}$.

Fraktion III, X, XI, XIII lieferten aus Alkohol unkrystallisiert Corydalin; Schmp. 134--1350.

Die Fraktionen II, IV, V, VI, VII, IX, XII und die Chloroformausschüttelung wurden gemeinsam in verdünnter Salzsäure gelöst, die Flülsigkeit (A) filtriert und etwas eingedampft. Nach längerem Stehen schieden sich knopfartig ausgebildete Krystalle aus, die aus Wasser umkrystallisiert als Nadeln erschienen. Diese wurden wieder in Wasser gelöst, die Lösung mit Natronlauge alkalisch gemacht und der entstandene Niederschlag aus Alkohol umkrystallisiert. Die rein weifs gefärbten Krystalle zeigten den Scbmelzpunkt $216^{\circ}-$ Cor y cavin.

Die Flülsigkeit A wurde gleichfalls mit Natronlauge versetzt, der Niederschlag abgesaugt und ausgewaschen. Beim Versuch, denselben aus Alkohol umzukrystallisieren, zeigte es sich, dals ein Teil von siedendem Alkohol leicht aufgenommen wurde, ein anderer schwer. Der erstere ergab nach mehrmaligem Umkrystallisieren reines Cor y da li n, der letztere wieder Corycavin.

In die auf verschiedene Weise, auch von Fraktion XII erhaltenen alkalischen Laugen wurde $\mathrm{CO}^{2}$ bis zur vollständigen Sättigung eingeleitet. Es entstand noch ein dicker, graugrüner Niederschlag Denselben mufste ich mehrmals wierler auflösen und fällen, bis or eine hellere Farbe angenommen hatte; dann wurde or abgesaugt, ausgewaschen und bei mälsiger Wärme getrocknet. Auch von diesem Niederschlag ging ein Teil leicht in Alkohol in Lösung und lieferte Bulbocapnin. Der Rückstand konute nur durch anhaltendes Kochen gelöst werden und ergab $\mathrm{C}$ o r y b u 1 b in Schmp. 2380, welches noch aus Chloroform umkrys tallisiert wurde. 
Ausbeute aus je $10 \mathrm{~kg}$ Knollen: Corydalin . . . $57 \mathrm{~g}$

Bulbocapnin . . 41 "
Corycavin . . 6 "
Corybulbin etwa 4 "

Das von D o b bi e und $L$ a $u$ der dargestellte Corytuberin und das amorphe Corydin habe ich aus den mir vorliegenden Corydalisknollen bisher nicht isolieren können.

$$
\begin{gathered}
\text { Corgdalin. } \\
\mathrm{C}_{22} \mathrm{H}_{27} \mathrm{NO}_{4} .
\end{gathered}
$$

Aus der Verschiedenheit der Angaben, welche eine grofse Anzahl von Forschern tiber das von ihnen aus den Knollen von Corydalis cava isolierte und mit dem Namen Corydal in bezeichnete Alkaloid machen, mufs man schlielsen, dafs dieselben nur ein Gemisch aus den verschiedenen Corydalisbasen vor sich gehabt haben. Erst A d e rman $\mathbf{n}^{\mathbf{1}}$ ) scheint ein ziemlich reines Corydalin dargestellt zu baben, das er allerdings nach seinen Beobachtungen für Hydroberberin oder einen hydroberberinartigen Körper hält und dem er daher die Formel $\mathrm{C}_{20} \mathrm{H}_{23} \mathrm{NO}_{4}$ zuerteilt.

Ad ermann beobachtete bei seinem Alkaloid auch dieselbe Gelbfärbung an Luft und Licht, beim Lösen in erwärmten Alkohol und beim Trocknen bei $100^{\circ}$, wie vorher schon $\mathrm{E}$. $S \mathrm{ch} \mathrm{midt}$, und kam zu der Annahme, dals Beziehungen desselben zum Berberin obwalten mülsten. Weiter stellte $\mathrm{Ad} \theta \mathrm{rm}$ an $\mathrm{n}$ aus seiner Base ein Goldsalz dar, welches denselben Au-Gehalt besitzt, wis ein von mir aus Alkohol umkrystallisiertes Corydalingoldchlorid.

B ir s ma $n^{2}$ ) schliefst sich in seinen Angaben über das Corydalin im wesentlichen den Ausführungen A dermann's an; auch er glaubt an eine Verwandtschaft des Corydalins und Berberins.

Eine umfassende Arbeit über das Corydalin haben alsdann Dob bie und $\mathrm{La}$ a $\mathrm{e}^{3}{ }^{3}$ ) im Jahre 1892 geliefert. Dieselben Forscher haben hierauf in den späteren Jahren weitere Veröffentlichungen über das Corydalin und seine Oxydationsprodukte, sowie über einige andere Corydalisalkaloide gemacht.

1) Inaug. Dissert. Dorpat 1890.

3) Inaug. Diss. Dorpat 1892.

3) Lond. Chem. Soc. 1892.

Arch. d. Pharm, CCXXXI v. Bds. 7. Heft. 
Diese Forscher glaubten zuerst, dafs dem bei $134,5^{0}$ schmelzenden Corydalin die Formel $\mathrm{C}_{22} \mathrm{H}_{28} \mathrm{NO}_{4}$ zuzuerteilen wäre, eine Formel, welche sie jedoch später in $\mathrm{C}_{22} \mathrm{H}_{29} \mathrm{NO}_{4}$ umgeändert haben. Die Resultate der Elementaranalysen von $\mathrm{D}$ ob bi $\theta$ und $L$ a u d er , die ich nachher übersichtlich mit den von Freund und Josephi und den von mir gefundenen zusammenstellen werde, berechtigen jedoch eher zu der Annahme, dals die Formel $\mathrm{C}_{22} \mathrm{H}_{27} \mathrm{NO}_{4}$ die richtigere ist. Auch bei der Analyse der Oxydationsprodukte ${ }^{1}$ ) des Corydalins, der Corydalinsäure und der Corydalsäure, haben diese Forscher in allen Fällen zu viol $\mathrm{C}$ und fast immor zu wenig $\mathrm{H}$ im Vergleich zu den berechneten Mengen gefunden.

Freund und $\mathrm{J}_{0 \mathrm{sephi}}{ }^{2}$ ) haben unabhängig von $\mathrm{D} \circ \mathrm{b}$ bi $\theta$ und $\mathrm{L}$ a u d er dem Corydalin vom Schmelzpunkt $134,5^{\circ}$ die Formel $\mathrm{C}_{22} \mathrm{H}_{27} \mathrm{NO}_{4}$ zuerteilt. Sie haben einige Salze dargestellt, jedoch das Studium desselben abgebrochen, als ihnen die Veröffentlichungen jener Forscher bekannt wurden.

Ich mufste mich, wenn ich die von $E . S \mathrm{ch}$ id t bereits im Jahre 1888 beobachtete eigentümliche Einwirkang des Jods anf das Corydalin, welche sonst von keinem der früheren Bearbeiter dieser Base in Betracht gezogen ist, näher studieren wollte, unter diesen Umständen naturgemäls zunächst mit dem Corydalin selbst, bez. mit der Feststellung seiner Molekularformel beschäftigen und zu diesem Zwecke auch die Darstellung und Analyse einiger Verbindungen desselben ausführen, ohne dabei jedoch irgendwie in das bisher von Dobbie und La uder bearbeitete Gebiet überzugreifen. Es konnte dies umsoweniger der Fall sein, als es sich in der nacbstehenden Arbeit nur um die Bestätigung und um die Weiterführung der bereits vor 8 Jahren von $\mathrm{Noelle}$ im hiesigen pharmazeutizchchemischen Institut mit Corydalin vom Schmelzpunkt $134,5^{0}$ angestellten Versuche handelt.

Sowohl das von Noelle stammende, als auch das von mir selbst dargestellte Corydalin bildet, aus Alkohol umkrystallisiert, durchsichtige, bis zu $5 \mathrm{~mm}$ grofse, prismatische Krystalle vom Schmelzpunkt 134-1350. Beim Auflösen in kaltem absoluten Alkohol bleibt dasselbe ungefärbt. Erst beim Erhitzen, namentlich wenn

1) Lond. Chem. Soc. 1893.

2) Annal. d. Chem. 277. 
letzteres bis zur Siedetemperatur fortgesetzt wird, nimmt die Lösung eine gelbe Farbe an, die bei längerem Kochen an Intensität zunimmt. Aus einer schwach gelb gefärbten, alkoholischen Lösung krystallisiert das Corydalin jedoch noch farblos aus. Erst aus den weiter eingeengten und dadurch intensiver gelb gewordenen Mutterlaugen schielsen gelbe Prismen an. Beim Stehen am Licht erleidet die alkoholische Lösung, ebenso wie die Krystalle selbst, eine intensive Gelbfärbung. Dieselben können zu analytischen $Z$ wecken nicht bei $100^{\circ}$ getrocknet werden, da sie auch hierbei eine gelbe Farbe annehmen, sondern müssen im Exsiccator, vor Licht gegchützt, zur Analyse vorbereitet werden. Aus den wässerigen Lösungen seiner Salze ist das Corydalin durch ätzende und kohlensaure Alkalien als rein weilses, krystallinisches Pulver abscheidbar. Im Ueberschufse dieser Fällungsmittel löst es sich nicht anf. Leicht löslich ist es in warmem Alkohol und in Chloroform.

Zur Elementaranalyse wurden nur rein weifse, grofs ausgebildete, bei $134,5^{0}$ schmelzende, im Exsiccator vor Licht geschïtzt getrocknete Krystalle verwendet.

I. Im beiderseits offenen Kupferoxydrohre mit vorgelegter reduzierter Kupferspirale verbrannt ergaben 0,2825 g Substanz $0,7424 \mathrm{~g} \mathrm{CO}_{2}$ und $0,1845 \mathrm{~g} \mathrm{H}_{2} \mathrm{O}$.

II. In gleicher Weise lieferten $0,2711 \mathrm{~g} \quad 0,7109 \mathrm{~g} \quad \mathrm{CO}_{2}$ und $0,1818 \mathrm{~g} \mathrm{H}_{2} \mathrm{O}$.

III. Im Kupferoxyd-Schnabelrohr ergaben 0,1925 $\mathrm{g}$ Corydalin $0,5033 \mathrm{~g} \mathrm{CO}_{2}$ und $0,1323 \mathrm{~g} \mathrm{H}_{2} \mathrm{O}$.

IV. Im beiderseits offenen, mit einem Gemisch von gekörntem Kupferoxyd und Bleichromat beschickten Rohr ergaben 0,2327 g Substanz $0,61 \cup 4 \mathrm{~g} \mathrm{CO}_{2}$ und $0,1500 \mathrm{~g} \mathrm{H}_{2} \mathrm{O}$.

V. In denselben Rohr lieferten $0,2055 \mathrm{~g}$ Substanz $0,5409 \mathrm{~g} \mathrm{CO}_{\mathbf{z}}$ und $0,1343 \mathrm{~g} \mathrm{H}_{2} \mathrm{O}$.

VI. Im Schnabelrohr, gefüllt mit einem Gemiscb von Kupferoxyd und Bleichromat, erhielt ich von $0,2444 \mathrm{~g}$ Substanz $0,6430 \mathrm{~g} \mathrm{CO}_{2}$ und $0,1710 \mathrm{~g}$ zu $\mathrm{H}_{2} \mathrm{O}$.

VII. 0,2416 g Substanz verbrauchten bei der N-Bestimmung nach W ill und Varrentrapp $6,8 \mathrm{ccm} 1 / 10 \mathrm{~N}$. HCl $=3,94$ Proz. N.

\begin{tabular}{|c|c|c|c|c|c|c|}
\hline \multicolumn{7}{|c|}{ Gefunden: } \\
\hline I. & II. & III. & IV. & V. & VI. & TII. \\
\hline C 71,64 & 71,44 & 71,27 & 71,50 & 71,77 & 71,64 & - \\
\hline H 7,25 & 7,45 & 7,63 & 7,16 & 7,26 & 7,77 & - \\
\hline $\boldsymbol{N} \quad-$ & - & - & - & - & - & 3,94 \\
\hline
\end{tabular}




$\begin{array}{cc}\text { Berechnet sind : für } \mathrm{C}_{22} \mathrm{H}_{27} \mathrm{NO}_{4} & \text { für } \mathrm{C}_{22} \mathrm{H}_{29} \mathrm{NO}_{4} \\ \mathrm{C}=71,54 & 71,16 \\ \mathrm{H}=7,32 & 7,82 \\ \mathrm{~N}=3,79 & 3,77\end{array}$

Zum Vergleich will ich die von Dobbie und La u der1), gowie die von Freund und $J o s e p h i^{2}$ ) gefundenen Werte hier anfübren.

Dobbie und Lauder.

Freund u. Josephi.

I. II. III. IV. V. VI. I. II.

$\begin{array}{llllllll}\text { C } 71,29 & 71,54 & 71,54 & - & 71,21 & 71,31 & 71,79 & 71,65\end{array}$

$\begin{array}{lllllllll}\mathrm{H} & 7,90 & 7,88 & 7,72 & 7,65 & - & 7,67 & 7,49 & 7,82\end{array}$

Ein Vergleich dieser Werte mufs ergeben, dafs die von $\mathrm{Dobbi \theta}$ und $\mathrm{Lauder}$ aufgestellte Formel $\mathrm{C}_{22} \mathrm{H}_{29} \mathrm{NO}_{4}$, selbst nachden Analysendaten dieser Forscher, die weniger wahrscheinliche ist. Ich neige mich daher der Ansicht zu, dafs dem Corydalin, entsprechend der Angabe von Freund und Josephi, die Formel $\mathrm{C}_{22} \mathrm{H}_{27} \mathrm{NO}_{4}$ zuzuerteilen ist, eine Formel, die ich durch die Analyse der durch Jod erhaltenen Einwirkungsprodukte bestätigt fand.

\section{Salze des Corydalins.}

Das Corydalin liefert mit starken Säuren im Allgemeinen gut charakterisierte Salze. Sonderbarer Weise ist es mir jedoch nicht gelungen, das salzsaure Salz desselben im krystallisierten Zustande darzustellen. Wenn ich nach Fround und Jose phi's Angaben ${ }^{3}$ ) die Base in salzsäurehaltigem Wasser löste und die klare Flürsigkeit auf dem Wasserbade eindampfte, resultierte ein gelblicher Firnis, der sich wohl wieder in warmem Wasser löste, beim Eindampfen jedoch, wie beim Verdunsten im Exsiccator immer nur eine zithe gelbliche Masse lieferte. Auch der Versuch, die Base in Alkohol zu lösen, Salzsäure bis zur schwach sauren Reaktion zuzufügen, und diese Lösung verdunsten zu lassen, ergab dasselbe Resultat.

Bromwasserstoffsaures Corydalin.

$\mathrm{C}_{22} \mathrm{H}_{27} \mathrm{NO}_{4}$. $\mathrm{HBr}$.

Das bromwasserstoffsaure Salz erhält man sowohl beim Auflösen der Base in verdünnter, erwärmter Bromwasserstoffsäure, als

1) Lon. Chem. Soc. 1892 .

2) Annal. d. Chemie 277.

3) Annal. d. Chemie 277 , p. 7. 
auch beim Zusatz derselben zu einer alkoholischen Lösung des Alkaloids bis zur schwach sauren Reaktion und Verdunsten der Lösungen. Aus heifsem Wasser krystallisiert es in rhombischen, lichtbrechenden Tafeln.

$0,2558 \mathbf{g}$ der Iufttrockenen Substanz verloren bei $10000,0009 \mathbf{g}$ $0,2549 \mathrm{~g}$ lieferten $0,1072 \mathrm{~g} \mathrm{Ag} \mathrm{Br}=17,84$ Proz. $\mathrm{Br}$

Berechnet für $\mathrm{C}_{22} \mathrm{H}_{27} \mathrm{NO}_{4}$. $\mathrm{H} \mathrm{Br}: \mathrm{Br}=17,77$ Proz.

Jodwasserstoffsaures Corydalin.

$\mathrm{C}_{22} \mathrm{H}_{27} \mathrm{NO}_{4}, \mathrm{HJ}$.

Dasselbe wird auf gleiche Weise erhalten, wie das bromwasserstoffsaure Salz. Aus heilsem Wasser schielsen lichtbrechende, rhombische Tafeln bis zu ca. $5 \mathrm{~mm}$ Grölse an, die getrocknet rein weifs erscheinen, beim Liegen an der Luft jedoch allmählich gelb werden.

0,2438 g verloren bei $100^{\circ}$ Nichta und ergaben $0,1137 \mathrm{~g} \mathrm{Ag} \mathrm{J}=25,38$ Proz. J. Berechnet für: $\mathrm{C}_{22} \mathrm{H}_{27} \mathrm{NO}_{4}, \mathrm{HJ}: \mathrm{J}=25,55$ Proz.

Corydalingitrat.

$\mathrm{C}_{22} \mathrm{H}_{27} \mathrm{NO}_{4}$. $\mathrm{HNO}_{3}$.

Das Nitrat erhielt ich durch vorsichtigen Zusatz von ver* dünnter Salpetersäure zu einer mälsig erwärmten, konzentrierten alkoholischen Auflösung der Base. Nach dem Erkalten und Stehenlassen schieden sich glänzende, tafelförmige, bei $198^{\circ}$ schmelzende Krystalle aus, die sich in heifsem Wasser schwer lösten. Dieselben erwiesen sich wasserfrei.

I. 0,2173 $\mathrm{g}$ Substanz ergaben bei der Elementaranalyse im Kupferoxydrohr 0,4866 g $\mathrm{CO}_{2}$ und $0,1256 \mathrm{~g} \mathrm{H}_{2} \mathrm{O}$.

II. $0,2070 \mathrm{~g}$ lieferten $0,4648 \mathrm{~g} \mathrm{CO}_{2}$ und $0,1208 \mathrm{~g} \mathrm{H}_{2} \mathrm{O}$.

III. $0,2042 \mathrm{~g}$ Substanz ergaben bei der Verbrennung in einem mit Kupferoxyd und Bleichromat beschickten Rohr $0,459 \mathrm{~g} \mathrm{CO}_{2}$ und $0,1212 \mathrm{~g} \mathrm{H}_{2} \mathrm{O}$.

Gefunden :

Berechnet für :

I. II. III. $\mathrm{C}_{22} \mathrm{H}_{27} \mathrm{NO}_{4}, \mathrm{HNO}_{3} \mathrm{C}_{22} \mathrm{H}_{29} \mathrm{NO}_{4}, \mathrm{HNO}_{3}$

C $61,05 \quad 61,20 \quad 61,21$

61,11

60.82

H $\quad 6,41 \quad 6,48 \quad 6,56$

6,48

6.91

Auch diese Werte berechtigen zu der Annahme, dafs dem Corydalin die Formel $\mathrm{C}_{22} \mathrm{H}_{27} \mathrm{NO}_{4}$ zukommt. 
Corydalingoldohlorid.

$$
\left(\mathrm{C}_{22} \mathrm{H}_{27} \mathrm{NO}_{4}, \mathrm{HCl}\right)_{2} \mathrm{AuCl}_{3} \text {. }
$$

Beim Zusatz von Goldchloridlösung im Ueberschufs zu einer stark salzsauren wässrigen Lösung des Corydalins fiel ein amorpher, hellgelber Niederschlag aus, den ich absetzen lies, absaugte und mit wenig Wasser nachwusch. In salzsäurehaltigem absoluten Alkohol löste sich derselbe beim Erwärmen zu einer gelbrot gefärbten Flüssigkeit auf und krystallisierte daraus in hellroten, zu Rosetten angeordneten Nadeln vom Schmelzpunkt 2070. Die zweite, durch Eindampfen der Lösung erhaltene Krystallisation lieferte gleichgeformte, nur etwas dunkler gefärbte Individuen.

Beim Trocknen bei $100^{\circ}$ verloren:

$$
\begin{aligned}
& 0,2411 \text { g Substanz } 0,0020 \mathrm{~g} \mathrm{H}_{\mathbf{8}} \mathrm{O} \\
& 0,2483 \text { " } 0,0023 \%
\end{aligned}
$$

Nach dem Trocknen hinterlielsen:

I. 0,2391 g Substanz 0,0424 g Au

II. $0,2460, \quad, 0,0442,$,

Ein normales Corydalingoldchlorid von der Formel

$$
\mathrm{C}_{22} \mathrm{H}_{27} \mathrm{NO}_{4} \mathrm{HCl}, \mathrm{Au} \mathrm{Cl} 3
$$

würde einen Goldgehalt von 27,74 Proz. erfordern; gefunden 17,75 Proz. und 17,96 Proz. Au.

Auch $\mathrm{A} d$ er m a n $\mathrm{n}^{1}$ ) hat aus seinem sogenannten hydroberberinartigen Körper, nach dem Umkrystallisieren aus Alkohol, ein Gold. doppelsalz von 17,71 Proz. Au-Gehalt dargestellt. Bei einem aus wässriger Lösung gefällten, rasch abgesaugten und getrockneten Präparat ermittelte or 25,15 Proz. Au. Ich erhielt bei einem auf gleiche Weise dargestellten Goldsalz ein solches von 24,03 Proz. Au, indem $0,235 \mathrm{~g}$ bei $100^{\circ}$ getrockneter Substanz $0,0572 \mathrm{~g}$ Au hinterliefsen.

Die Uebereinstimmung der von $\mathrm{Ad}$ ermann und mir ausgeführten Analysen, des aus Alkohol umkrystallisierten und auch des aus wässriger Lösung gefällten Golddoppelsalzes des Corydalins lassen schliefsen, dafs beim Zusatz von Goldchlorid zu wässriger, salzsäureenthaltender Corydalinlösung wohl zunäehst ein normales Corydalingoldchlorid abgeschieden wird, welches aber sehr bald

1) Inaug. Dissert. Dorpat 1890. 
eine Veränderung erleidet und durch Umkrystallisieren aus Alkohol in eine Verbindung von anormaler, aber konstanter Zusammensetzung übergeht.

Die von mir weiterhin ausgefübrten Elementaranalysen und Culorbestimmungen lieferten den Beweis, dals dem aus Alkohol umkrystallisierten Corydalingoldchlorid die Formel

$$
\left(\mathrm{C}_{22} \mathrm{H}_{27} \mathrm{NO}_{4} \cdot \mathrm{H} \mathrm{Cl}\right)_{2}, \mathrm{Au} \mathrm{Cl}_{3}
$$

zukommt. Zur Ausführung der Analysen stellte ich grölsere Mengen dieses Doppelsalzes in oben beschriebener Weiso dar und ermittelte zunächst wieder den Au-Gehalt.

III. $0,2029 \mathrm{~g}$ verloren bei $100^{\circ} 0,0021 \mathrm{~g} \mathrm{H}_{2} \mathrm{O}$ und lieferten $0,0356 \mathrm{~g}$ $\Delta \mathfrak{u}=17,72$ Proz. Au.

IV. Bei der Elementaranalyse im Bleichromatrohre ergaben $\mathbf{0 , 2 0 3 4} \mathbf{g}$ nach dem Trocknen bei $100^{\circ} 0,354$ g CO$~_{2}$ und $0,0894 \mathbf{g ~ H}_{2} \mathrm{O}$ oder 47,44 Proz. C und 4,88 Proz. H.

Die Chlorbestimmungen versuchte ich zunächst nach der allgemein üblichen Methode durch Glühen der mit chlorfreiem trocknem Natriumcarbonat gemischten Substanz unter einer Decke von Natrium. carbonat bis zur vollständigen Zerstörung der organischen Substanz auszufübren. Ich erhielt jedoch Werte, welche mit den berechneten nicht übereinstimmten.

$0,2448 \mathrm{~g}$ bei $100^{0}$ getrocknet ergaben $0,1337 \mathrm{~g} \mathrm{Ag} \mathrm{Cl}=13,48$ Proz. Cl.

$0,2386 \mathbf{g}$ getrockneter Substanz lieferten $0,1300 \mathrm{~g}$ Ag Cl= 13,47 Proz. Cl.

Gluhte ich die Mischung des Goldsalzes mit dem Natriumcarbonat nur schwach, so dals die organische Substanz noch kohlig zurückblieb, so erbielt ich folgendes Resultat:

$0,2884 \mathrm{~g}$ getrockneter Substanz ergaben $0,1704 \mathrm{~g} \mathrm{Ag} \mathrm{Cl}=$ 14,59 Proz. Cl.

Es schien somit beim Githen mit Natriumcarbonat ein Teil des Chlors verloren zu gehen. Ich kochte daher eine abgewogene Menge mit einer konzentrierten Lösung reinen Natriumcarbonats etwa fünf Minuten, bis sich das Gold als bra unschwarzerNiederschlag ausgeschieden hatte. Ich fand hierbei

V. aus $0,2264 \mathrm{~g}$ getrockneter Substanz $0,1535 \mathrm{~g} \quad \mathrm{AgCl}=$ 16,75 Proz. Cl.

Das Resultat der Analysen war demnach folgendes:

I. II. III. IV. V.

$\begin{array}{lcccccc}\mathrm{An} & 17,75 & 17,96 & 17,72 & - & - \\ \mathrm{O} & - & - & - & 47,44 & - \\ \mathrm{H} & - & - & - & 4,88 & - \\ \mathrm{Cl} & - & - & - & - & 16,75 .\end{array}$


H. Ziegenbein: Alkaloide von Corydalis cava.

Berechnet für :

$\begin{array}{cc}\left(\mathrm{C}_{23} \mathrm{H}_{27} \mathrm{NO}_{4} \cdot \mathrm{H} \mathrm{Cl}\right)_{2}, \mathrm{Au} \mathrm{Cl} & \mathrm{C}_{22} \mathrm{H}_{27} \mathrm{NO}_{4} \mathrm{H} \mathrm{Cl}, \mathrm{Au} \mathrm{Cl} \\ 17,74 & 27,75 \\ 47,39 & 37,25 \\ 5,02 & 3,75 \\ 16,82 & 20,03\end{array}$

Oxydationdes Corydalins in heisser alkalischer Lösung.

Da ein Vorversuch gelehrt hatte, dafs bei gewöhnlicher Temperatur die Oxydation des Corydalins in alkalischer Lösung nur sehr langzam fortschreitet, löste ich $1 \mathrm{~g}$ Corydalin mit wenig verdünnter Schwefelsäure zu $100 \mathrm{ccm}$ aut, machte diese Lösung mit Barytwasser schwach alkalisch, erwärmte auf dem Wasserbade und fügte Baryumpermanganatlösung (4:1000), bis die Rotfärbung einige Zeit bestehen blieb. Ich verbrauchte hierzu 2,3 $\mathrm{g} \mathrm{Ba} \mathrm{Mn}_{2} \mathrm{O}_{8}$. In dem Manganniederschlage konnte ich viel $\mathrm{CO}_{2}$ und nur wenig Oxalsäure nachweisen. Die gelbe, wässerige Lösung färbte sich beim Eindampfen dunkler, auch schieden sich an den Wandungen des Gefälses braune Massen aus. Die Ausschüttelung der filtrierten Lösung mit Aether ergab, nach dem Verdunsten desselben, einen reichlichen, in Wasser löslichen Rückstand. Die wässerige, mit Ammoniak alkalisch gemachte Lösung desselben wurde durch Bleiessig grau-weifs gefällt. Diesen Niederschlag suspendierte ich behufs weiterer Reinigung in Wasser, leitete in die Mischung, ebenso wie in die wässerige Flüssigkeit, Schwefelwasserstoff ein, filtrierte das ausgeschiedene $\mathrm{PbS}$ ab und verjagte den $\mathrm{H}_{2} \mathrm{~S}$ durch Eindampten auf ein kleines Volumen. Die Flüssigkeit schüttelte ich nochmals mit Aether aus, löste den Verdunstungsrückstand in wenig Wasser und überliels die Lösung der Ruhe im Exsiccator. Nach einigen Tagen scbieden sich Krystalle ab, die, nach einmaligem Umkrystallisieren, die Form, den Schmelzpunkt und die Reaktion der He mi p in s äu re zeigten. Da zu derselben Zeit, als ich diesen Versuch ausgefülrt hatte, D ob bi e und L a u d e r ${ }^{1}$ ) veröffentlichten, dafs sie bei der Oxydation der Corydalinsäure Hemipinsäure erhalten hätten, habe ich die weiteren Versuche aufgegeben. Bemerkenswert ist aber, dals das Corydalin,

1) Chem. Ztg. 189t, p. 195 t. 
ebenso wie das Berberin, bei der Oxydation mit Baryumpermanganat Hemipinsäure liefert.

Einwirkung von Jod auf Corydalin.

Eine kalte alkoholische Corydalinlösung versetzte ich so lange tropfenweise mit alkoholischer Jodlösung, bis die braune Färbung nicht mehr in eine gelbe überging, sondern bestehen blieb. Nach dem Eindampfen schied sich aus dieser Füssigkeit ein Gemisch von gelben und weifsen Krystallen ab, ein Beweis dafür, dals nur ein Teil des Corydalins verändert war. Auch wenn ich eine erwärmte Corydalinlösung in gleicher Weise mit Jodlösung behandelte, zeigten sich nach dem Erkalten, neben vielen gelben Nadeln weisse Krystalle. Ich verfuhr daher in derselben Weise wie $\mathrm{E}$. S $\mathrm{chmidt}$ ) beim Canadin, indem ich Jod bei $100^{\circ}$ unter Druck einwirken liels.

$0,2109 \mathrm{~g}$ im Exsiccator getrocknetes Corydalin wurden in $30 \mathrm{ccm}$ 96 Proz. Alkohols gelöst, $0,9354 \mathrm{~g}$ redestillierten, grofsblätterigen, über Aetzkalk sorgfältig ausgetrockneten Jods hinzugefügt und die Lösung drei Stunden lang in einer gut schliefsenden Drucklasche im Wasserbade erwärmt. Nach der Einwirkung sah die Flüssigkeit noch braun aus; am Boden der Flasche hafteten braunschwarze, zusammengeballte Massen von Perjodiden. Nach dem Erkalten wurden ca. $2 \mathrm{~g}$ KJ, zerriebenes Natriumbikarbonat bis zur schwach alkalischen Reaktion und $60 \mathrm{ccm}^{1}{ }_{110} \mathrm{~N} . \mathrm{Na}_{2} \mathrm{~S}_{2} \mathrm{O}_{\mathrm{a}}$-lösung zugefügt und die Mischung mehrere Tage lang auf dem Wasserbade gelinde erwärmt. Nie Flüssigkeit färbte sich hierdurch sehr bald gelb, auch die schwarzen Perjodide wurden allmälhich zerlegt, sodal's schliefslich eine gelbe Lösung resultierte, in der feine, gelbe Nadeln suspendiert waren. Die ganze Mischung wurde nun in einen $500 \mathrm{ccm}$-Kolben gebracht, sorgfältig nachgespulalt und zur Marke aufgefüllt. Der Ueberschufs von $1 / 10 \mathrm{~N} . \mathrm{Na}_{2} \mathrm{~S}_{2} \mathrm{O}_{3}$ wurde mit $1 / 10 \mathrm{~N}$. J zurücktitriert, unter Anwendung von Stärke als Indikator. $J_{e} 200 \mathrm{ccm}$ brauchten $2,50 \mathrm{ccm} 1 / 10 \mathrm{~N} . J, 500 \mathrm{~cm}$ also $6,25 \mathrm{ccm} 1 / 10 \mathrm{~N}$. J mithin waren zur Bindung des überschüssig zugesetzten Jods $60-6,25=53,75 \mathrm{~cm}{ }^{1} 10 \mathrm{~N} . \mathrm{Na}_{2} \mathrm{~S}_{2} \mathrm{O}_{3}$ nötig gewesen. Oder da $1 \mathrm{ccm}$ $1 / 10 \mathrm{~N} \cdot \mathrm{Na}_{2} \mathrm{~S}_{2} \mathrm{O}_{3}=0,0127 \mathrm{~g} \mathrm{~J}$, so waren $53,75.0,0127=0,6826 \mathrm{~g} \mathrm{~J}$ noch frei vorhanden, während $0,9354-0,6826=0,2528 \mathrm{~g} \mathrm{~J}$, enteprechend 4 Atomen, auf das Corydalin eingewirkt hatten. Der Vorgang dürfte durch folgende Gleichung zu illustrieren sein:

$$
\begin{gathered}
\mathrm{C}_{22} \mathrm{H}_{27} \mathrm{NO}_{4}+4 \mathrm{~J}=\mathrm{C}_{22} \mathrm{H}_{23} \mathrm{NO}_{4}, \mathrm{HJ}+3 \mathrm{HJ} \\
369: 5,08=0,2109: \mathrm{x} \\
\mathrm{x}=0,2904 \mathrm{~g} J .
\end{gathered}
$$

1) Archiv d. Pharm. 1894, p. 144. 
Nach obiger Gleichung hätten also 0,2901 g J auf 0,2109 $\mathrm{g}$ Corydalin einwirken müssen:

Ein zweiter, in ganz gleicher Weise angestellter Versuch verlief folgendermafue :

$0,7201 \mathrm{~g}$ Corydalin wurden mit $4,0327 \mathrm{~g} \mathrm{~J}$ und $120 \mathrm{ccm}$ Alkohol erhitzt, $2 \mathrm{~g}$ KJ, Natriumbikarbonat und $300 \mathrm{ccm} 1 / 10 \mathrm{~N} . \mathrm{Na}_{2} \mathrm{~S}_{2} \mathrm{O}_{3}$ zugefügt, und die Mischung zu $1000 \mathrm{ccm}$ aufgefüllt. Je $200 \mathrm{ccm}$ verbrauchten $11 \mathrm{ccm} 1 / 20 \mathrm{~N}$.J, $1000 \mathrm{ccm}$ also $55 \mathrm{ccm} \mathrm{1/10} \mathrm{N.J.} \mathrm{Es} \mathrm{waren}$ somit $245 \cdot 0,0127=3,1115 \mathrm{~g} \mathrm{~J}$ vicht, $4,0327-3,1115=0.9212 \mathrm{~g} \mathrm{~J}$ hingegen in Reaktion getreten. Berechnet wären, unter Zugrundelegung obiger Gleichung, $0,9910 \mathrm{~g} \mathrm{~J}$.

Dafs der unter diesen Bedingungen gebildete neue Körper dem Berberin sehr nahe verwandt ist, geht schon aus der Bildungsweise und der intensiv gelben Farbe desselben hervor. Es mag diese neue Verbindung vorlänfig als "D e h y drocory d a li n " bezeichnet werden.

Sowohl die zur Titration gebrauchton Flüssigkeiten, als auch die noeh klaren gelben Lösungen verwendete ich zur Reindarstellung des jodwasserstoffsauren Dehydrocorydalins, soweit dieses nicht schon auskrystallisiert und abfiltriert war. Die gesamten Flüssigkeitsmengen damptte ich zu diesem Zwecke zur Trockne ein und extrahierte den Rückstand mit heifsem, 96 prozentigen Alkohol. Das jodwasserstoffsaure Salz ist in kaltem Alkohol schwer löslich und krystallisiert daher sehr rasch in hellgelben, kleinen glänzenden Nadeln aus:

Um gröfsere Mengen Debydrocorydalinhydrojodids darzustellen verfuhr ich in ähnlicher Weise, wie bei den quantitativen Versuchen, und zwar wendete ich hauptsächlich dasjenige Corydalin an, welches wohl den richtigen Schmelzpunkt $134,5^{0}$ zeigte, aber schon etwas gelb gefärbt war. Je $5 \mathrm{~g}$ davon erhitzte ich mit $10 \mathrm{~g}$ Jod und 200 cem Alkohol unter Druck, fügte nach dem Erkalten Natriumbicarbonat und Natriumthiosulfat in wässriger Lösung zu, trennte jedoch nach etwa eintägigem Erwärmen die gelb gewordenen, warmen alkoholischen Lösungen von den Perjodiden, weil aus ersteren nach dem Abfiltrieren und Erkalten sofort das jodwasserstoffsaure Salz auskrystallisierte und beim weiteren Eindampfen in noch grölserer Menge rein zu gewinnen war. Die Perjodide erwärmte ich in einer Schale mit Natriumbicarbonat und -thiosulfat. unter Zusatz von Wasser und wenig Alkohol so lange, bis die 
ganze Masse rein gelb geworden war, verdampfte dann den überschüssigen Alkobol, filtrierte die wässrige Lösung, welche die Hauptmenge der organischen Salze enthielt, ab, wusch den Rückstand noch mehrmals mit Wasser nach und kochte ihn schliefslich wiederholt mit 96 prozentigem Alkohol aus. Die gewounene Menge trennte ich durch Absaugen von der Mutterlange, krystallisierte sie aus Alkohol nochmals um, prefste und trocknete sie bei gewöhnlicher 'Temperatur.

Das so erhaltene jodwasserstoffsaure Dehydrocorydalin stellt, mit der Yupe betrachtet, kleine, hellgelbe, glänzende, häufig zu Rosetten angeordnete Nadeln dar, welche unter dem Mikroskop als durchscheinende, prismatische Säulen sich kennzeichnen. Dieselben sind lichtbestündig, lösen sich in heifsem Alkohol verhältuismälsig leicht, in kaltem schwer, in heifsem Wasser sehr schwer und in kaltem fast gar nicht auf.

Das jodwasserstoffsaure Dehydrocorydalin erwies sich als. krystallwasserhaltig.

I. 0,9534 $g$ Substanz verloren nach mehrtägigem Trockn! im Wassertrockenschrank $0,0619 \mathrm{~g} \mathrm{H}_{2} \mathrm{O}=6,49$ Proz. $\mathrm{H}_{2} \mathrm{O}$.

II. $0.2186 \mathrm{~g}$ über $\mathrm{H}_{2} \mathrm{SO}_{4}$ getrocknet verloren Nichts.

Dieselbe Menge ergab bei der direkten Jodbestimmung, Zutügen von Silbernitrat im Ueberschuf $f_{s}$ zu der kochenden, mit wenigen Tropfen Salpttersäure angesäuerten wässrigen Lösung, 0,996; g Ag J. Berechnet für:

$$
\begin{array}{cccc}
\mathrm{C}_{22} \mathrm{H}_{23} \mathrm{NO}_{4}, \mathrm{H} \mathrm{J}+2 \mathrm{H}_{2} \mathrm{O} & \mathrm{I} & \text { II. } \\
\mathrm{H}_{2} \mathrm{O} & =6.80 \text { Proz. } & 6.49 & - \\
\mathrm{J} & =24,00 \quad & - & 23,78 .
\end{array}
$$

Gefunden:

Es dürtte demnach dem jodwasserstoffsauren Dehyluocorydalin die Formel $\mathrm{C}_{22} \mathrm{H}_{23} \mathrm{NO}_{4}$, $\mathrm{HJ}+2 \mathrm{H}_{2} \mathrm{O}$ zukommen.

Salzsaures Dehydrocorydalin.

$$
\mathrm{C}_{23} \mathrm{H}_{23} \mathrm{NO}_{4}, \mathrm{H} \mathrm{Cl}+4 \mathrm{H}_{2} \mathrm{O} \text {. }
$$

Das Hydrochlorid lälst sich leicht aus dem Hydrojodit orbalten, indem man letzteres in verdünnt-alkoholischer Lösung unter Zusatz von Salzsäure mit feuchtem Chlorsilber längere Zeit kocht. Es scheiden sich nach dem Abfiltrieren und Erkalten gelbe Blättchen und Säulen ab, die allmählich dadurch dunkler erscheinev, dås sie sich übereinander schichten. Die Krystalle sind in Wasser und Alkohol leicht löslich. 
$0,5819 \mathrm{~g}$ verloren bei $100^{0} 0,0914 \mathrm{~g} \mathrm{H}_{2} \mathrm{O}$.

Bei $105^{0}-110^{\circ}$ verloren sie $0,1128 \mathrm{~g}=22,03$ Proz. und wurden braun. Es scheint demnach boi dieser Temperatur eine teilweise Abspaltung ron $\mathrm{B} \mathrm{Cl}$ einzutreten.

Berechnet fur: $\mathrm{C}_{23} \mathrm{H}_{23} \mathrm{NO}_{4}, \mathrm{H} \mathrm{Cl}+4 \mathrm{H}_{2} \mathrm{O}$ $\mathrm{H}_{2} \mathrm{O}=15,20$ Proz.

Gefunden: $\mathrm{H}_{2} \mathrm{O}=15,70$,

$0,2917 \mathrm{~g}$ bei $100^{\circ}$ gitrockneter Substanz lieferten nach Carius 0,1027 g Ag Cl $=8,70$ Proz. Cl.

Berechnet : 8,84 Proz. Cl.

Debydrocorydalingoldehlorid.

$$
\mathrm{C}_{22} \mathrm{H}_{23} \mathrm{NO}_{4} \mathrm{HCl}, \mathrm{Au} \mathrm{Cl}_{3} \text {. }
$$

Eine Probe der wässrigen Lösung des salzsauren Salzes gab mit Goldchlorid eine ähnliche braunrote Fällung wie das Berberin. Ich versetzte daher eine gröfsere Menge der wässrigen Lösung des salzsauren Salzes, nach starkem Ansänern mit HCl, mit Goldchlorid im Ueberschula. Den sich ausscheidenden braunen Niederschlag sangte ich ab, wusch ihn aus und löste ihn durch anbaltendes Kochen mit angesänertem absoluten Alkohol, in dem er sehr schwer löslich war. Nach dem Erkalten schieden sich kleine, rotbraune Nadeln aus, die in Farbe und Gestalt durchaus denen des Berberingoldcblorids glichen. Der Schmelzpunkt wurde bei $219^{\circ}$ ermittelt.

$0,2081 \mathrm{~g}$ verloren bei $100^{\circ}$ getrocknet Nichts und hinterliefsen 0,0578 g $A \mathbf{u}=27,77$ Proz. Au.

Berechnet für $\mathrm{C}_{22} \mathrm{H}_{23} \mathrm{NO}_{4} . \mathrm{HCl}, \mathrm{Au} \mathrm{Cl} \mathrm{Cl}_{3}: \mathrm{Au}=27,90$ Proz.

Dehydrocorydalinplatinchlorid.

$$
\left(\mathrm{C}_{22} \mathrm{H}_{23} \mathrm{NO}_{4}, \mathrm{HCl}\right)_{2} \mathrm{Pt} \mathrm{Cl}_{4}+6 \mathrm{H}_{2} \mathrm{O} \text {. }
$$

Beim Versetzen einer stark salzsauren wässrigen Lösung des Dehydrocorydalinhydrochlorids mit Platinchlorid im Ueberschufs, scheidet sich ein flockiger, gelblichweifser Niederschlag ab. Derselbe ist abzusaugen, mit Wasser nachzuwaschen und in absolutem, stark angesäuerten Alkohol zu lösen. Das in der Kälte auskrystallisierendo Doppelsalz bildet schöne, hellgelbe, bis $5 \mathrm{~mm}$ lange Nadeln. 0,2346 g Substanz verloren bei $100^{\circ} 0,0206$ g H$_{2} \mathrm{O}=8,78$ Proz. $\mathrm{H}_{2} \mathrm{O}$.

Dieser Wert würde einem Krystallwassergehalt von 6 Molekülen entsprechen, denn die Forrael $\left(\mathrm{C}_{22} \mathrm{H}_{23} \mathrm{NO}_{4} \mathrm{HCl}\right)_{2} \mathrm{PtCl}_{4}+6 \mathrm{H}_{2} \mathrm{O}$ verlangt 8,31 Proz. $\mathrm{H}_{22} \mathrm{O}$. 
Bei der Platinbestimmung blieben zurüek aus 0,214 " $\mathrm{g}$ bei $100^{\circ}$ getrockneter Subst. 0,037 g Pt $=19,29$ Proz. Pt.

$$
\begin{array}{cc}
\text { Berechnet: } & \text { Gefunden: } \\
\mathrm{H}_{3} \mathrm{O}=8,61 \text { Proz. } & 8,78 \text { Proz. } \\
\mathrm{Pt} & =17,21 \%
\end{array}
$$

Aus dem jodwasserstoff- und chlorwasserstoffsauren Salze suchte ich die freie Base dadurch darzustellen, dafs ich zu Lösungen derselben Kalilauge, Natriumcarbonatlösung und Ammoniak fügte. Es trat jedoch keinerlei Fällung ein; ebensowenig konnte ich durch Ausschütteln mit Chloroform und Aether das freie Debydrocorydalin isolieren.

Der berberinartige Charakter des Dehydrocorydalins lies jedoch erwarten, dals dasselbe, obenso wie das Berberin'1) eine Acetonverbindung geben würde, aus der dann event. die freie Base, andere Salze und Verbindungen gewonnen werden konnten. Ein Versuch bestätigte diese Annahme.

Darstellung einer Acetonverbindung des Dehydrocorydalins.

Je $5,0 \mathrm{~g}$ des jodwasserstoffsauren Dehydrocorydalins wurden: in einem Rundkolben mit $100 \mathrm{~g}$ Wasser und $50 \mathrm{~g}$ Aceton am Rüickflufskühler auf der Asbestpappe so lange gekocht, bis vollständige Klärung eingetreten war, die Lösung filtriert und mit starker Natronlauge alkalisch gemacht. Alsbald trübte sich die Flässigkeit und allmählich schieden sich grofse, ölige Tropfen ab, die nach eintägigem Stehen erstarrt waren. Dieselben wurden von der Mutterlauge getrennt, mit Wasser so lange nachgewaschen, his dasselbe alkalisch reagierte, und zwischen Flielspapier getrocknet. Aus den Mutterlaugen konnten nur coch ganz geringe Mengen öliger Tropfen durch Eindamplen gewonnen werden. Die gepressten erstarten Tröpfchen stellten aufsen hellgelb, innen braun gefärbte Massen dar, die zum weiteren Trocknen zerrieben und in einen vor Licht geschützten Schwefelsäure-Exsiccator gebracht wurden. Das. anfänglich dunkelgelb gefärbte Pulver nahm allmählig eine hellere Farbe an. Um die wahrscheinlich vorliegende Acetonverbindung. in eine analysierbare Form zu bringen, versuchte ich, dieselbe ais verschiedenen Lösungsmitteln umzukrystallisieren. Eine kleine Probe

1) Arch. d. Pharm. 1890 p. 607. 
des vollständig trocknen, hellgelben Pulvers löste ich zu diesem Zwecke durch ganz vorsichtiges Erwärmen in starkem Alkohol und setzte tropfenweise Wasser bis zur eben wieder verschwindenden Trübung zu: nach dem Stehen schieden sich gelbe Krystalle aus. Als jedoch dieser Versuch mit grölseren Mengen anscheinend unter denselben Kautelen wiederholt wurde, wollte es nicht gelingen, jene Krystalle wieder zu erhalten. Auch Aufösen in reinem und in verdünntem Aceton tührten zu keinem Ziele. Ich mufs es deshalb späteren Versuchen überlassen, diese Verbindung in krystallisierter Gestalt zu gewinnen. Dals jedoch unter den obigen Bedingungen aus dem Dehydrocorydalin eine Acetonverbindung gebildet ist, dürfte keinem Zweifel unterliegen. Denn einmal spricht die dem AcetonBerberin analoge Bildungsweise und Form dafür, und ferner der Umstand, dafs beim Erwärmen mit verdünnten Mineralsäuren, Aceton abgespalten wird, unter gleichzeitiger Bildung der entsprechenden Salze des Dehydrocorydalins.

Versuch, das freie Dehydrocorydalin darzustellen. $\mathrm{C}_{\mathrm{az}} \mathrm{z}^{\prime} \mathrm{s}^{1}$ ) Angaben über die Darstellung von reinem Berberin aus Aceton-Beberin folgend, erhitzte ich $1,0 \mathrm{~g}$ der Acetonverbindung mit $5 \mathrm{ccm}$ Chloroform und $50 \mathrm{ccm}$ absolutem Alkohol 12 Stunden lang auf dem Wasserbade am Rückflusskühler, destillierte dann einen Teil der gelb gefärbten Flüfsigkeit $a \bar{b}$, wobei sie einen braunen Farbenton annahm, und überliefs den Rest, geschützt vor Licht, der freiwilligen Verdunstung. In der Mitte der benutzten Glasschale setzten sich gelbe Körner an, die von einem braunen Rand umgeben waren. Letzterer konnte leicht durch Abspülen mit Essigäth ir entfernt werden, woraut die gelben Krystalle in Wasser gelöst wurden. Beim Verdunstenlassen im Exsiccator schieden sich gleichgeformte gelbe Krystalle ab, doch war die Menge derselben bis jetzt noch so gering, dals sie bisher keiner Analyse unterworfen werden konnte.

Salzsaures Dehydrocorydalin aus der Acetonverbindung.

Durch Suspendieren der Acetonverbindung in Wasser, Erwarmen der Mischung und tropfenweisen Zusatz von verdünnter 1) Arch. d. Pherm. 1890 p. 609. 
Salzsäure bis zur schwach sauren Reaktion, tritt unter Abgabe von Aceton vollständige Lösung zu einer hellgelb gefärbten Flüssigkeit ein. Nach dem Eindampfen krystallisiort das salzsaure Salz in kleinen, gelbroten,büschelförmig angeordneten Nadeln. Unter dem Mikroskop erscheinen sie als dünne, geschichtete Tafeln. Der Schmelzpunkt des aus Wasser nochmals umkrystallisierten Salzes wurde bei $129^{\circ}$ ermittelt.

Da ich die Beobachtung gemacht hatte, dafs beim Trocknen bei $100^{\circ}$ während einigen Stunden das Krystallwasser wohl vollständig abgegeben wird, hingegen beim weiteren Stehenlassen im Trockenschranke das Gewicht von Tag zu Tag um $1 \mathrm{mg}$ abnimmt, mufste ich annehmen, dals hierbei Abspaltung eines Teiles der flüchtigen Säuren eintritt. Ich trocknete daher von jetzt an die meisten Salze über Schwefelsäure.

I. $0,2258 \mathrm{~g}$ des zerriebenen Salzes gaben über Schwefelsäure bei circa 14 tägigem Stehen bis zum konstanten Gewicht ab $0,034 \mathrm{~g}$ $=15,05$ Proz. $\mathrm{H}_{2} \mathrm{O}$.

II. $0,1918 \mathbf{g}$ derartig getrockneter Substanz lieferten $0,0705 \mathbf{g}$ $\mathrm{Ag} \mathrm{Cl}=9,01$ Proz. Cl. Berechnet 8,84 Proz. Cl.

Ich analysierte noch ein krystallwasserhaltiges Salz und erhielt dabei folgenden Wert:

III. 0,2177 g Substanz 0,066 g Ag Cl $=7,45$ Proz, Cl.

Berechnet für $\mathrm{C}_{22} \mathrm{H}_{28} \mathrm{NO}_{4} \mathrm{BCl}+4 \mathrm{H}_{2} \mathrm{O}: \mathrm{Cl}=7,49$ Proz.

Einen Teil des salzsauren Salzes krystallisierte ich aus Alkohol um and machte dabei die Beobachtung, dafs es in diesem Lösungsmittel etwas schwerer löslich ist als in Wasser. Auch schien dio Krystallform eine andere zu sein, indem sich zunächst feine, hellgelbe Nadeln, später bräunliche, langgestreckte Tafeln ausschieden, die sich an und uber einander legten. Unter dem Mikroskop betrachtet, erschienen gie als dünne Säulen oder Blättchen, die sich za sehr vielen übereinander geschichtet hatten.

IV. $0,2027 \mathrm{~g}$ zerriebene Substanz erlitten uber Schwefelsăure einen Gewichtsverlust von $0,0299 \mathrm{~g}=14,70$ Proz. $\mathrm{H}_{2} \mathrm{O}$.

V. 0,1728 g getrocknete Substanz lioferten $0,0631 \quad \mathbf{g ~ A g ~ C l}$ $=9,02$ Proz. Cl.

Im Hinblick auf diese Analysendaten mufs man annehmen, dafs das aus wässriger Lösung gewonnene Salz identisch mit dem aus Alkohol umkrystallisierten ist, und dals beiden die Formel

zukommat.

$$
\mathrm{C}_{22} \mathrm{H}_{23} \mathrm{NO}_{4}, \mathrm{HCl}+4 \mathrm{H}_{2} \mathrm{O}
$$


Es zeigt sich aber auch fernerbin, dals das mit Hilfe der Acetonverbindung gewonnene Salz dieselben Eigenschaften hat, wie das durch Umsetzen des Hydrojodids mit Chlorsilber erhaltene.

$\begin{array}{cccc}\text { Berechnet } & \begin{array}{c}\text { Hydrochlorid } \\ \text { aus Hydrojodid }\end{array} & \begin{array}{c}\text { Hydrochlorid } \\ \text { aus Acetonverbdg. } \\ \text { umk. aus } \mathrm{H}_{2} \mathrm{O}\end{array} & \begin{array}{c}\text { Hydrochlorid } \\ \text { aus Acetonverbdg. }\end{array} \\ \begin{array}{cccc}\text { umk. aus } \mathrm{C}_{2} \mathrm{H}_{5} \mathrm{OH} \\ \mathrm{H}_{2} \mathrm{O}=15,20\end{array} & 15,70 & 15,05 & 14,70 \\ \mathrm{Cl}=8,84 & 8,70 & 9,01 & 9,02\end{array}$

Bromwasserstoffsaures Dehydrocorydalin

$$
\mathrm{C}_{22} \mathrm{H}_{23} \mathrm{NO}_{4} \mathrm{HBr}+4 \mathrm{H}_{2} \mathrm{O} \text {. }
$$

Dasselbe wird analog dem Chlorbydrat aus der Acetonverbindung durch Erwärmon derselben mit verdünnter Bromwasserstoffsäure bis zum Verschwinden des Acetongeruches dargestellt. Dasselbe ist schwerer löslich in Wasser als das salzsaure Salz und krystallisiert daraus in gelbbraunen Nadeln, die beim Stehen am Licht eine gelb-grüne Farbe annehmen. Die lufttrockene Substanz sintert bei $126^{\circ}$ zusammen, um bald darauf zu öligen, braunen Tröpfchen zu schmelzen.

Aus Alkohol umkrystallisiert, scheiden sich, ähnlich wio beim salzsauren Salz, erst hellgelbe Nadeln, beim längeren Stehen Tafeln und Säulen von dunklerer Farbe ab. Die Analyse des aus Wasser umkrystallisierten Salzes ergab folgende Werte:

I. $0,259 \mathrm{~g}$ zerriebene Substanz verloren über Schwefelsäure $0,0366 \mathrm{~g}=14,13$ Proz. $\mathrm{H}_{2} \mathrm{O}$.

II. $0,2224 \mathrm{~g}$ getrocknete Substanz lieterten $0,0941 \mathrm{~g}$ Ag Br $=$ 17,98 Proz. Br. Daten :

Das aus alkoholischer Lösung gewonnene Salz lieferte folgende

III. $0,2603 \mathbf{g}$ erlitten über Schwefelsäure einen Wasserverlust von $0,0391 \mathrm{~g}=15,02$ Proz. $\mathrm{H}_{3} \mathrm{O}$

IV. $0,2212 \mathrm{~g}$ getrocknetes Salz ergaben $0,093 \mathrm{~g} \mathrm{Ag} \mathrm{Br}=$ 17,85 Proz. Br.

Berechnet für:

$\mathrm{C}_{22} \mathrm{~B}_{23} \mathrm{NO}_{4} \mathrm{H} \mathrm{Br}$

$\mathrm{Br}=17,93$ Proz.

Berechnet fur:

$\mathrm{C}_{22} \mathrm{H}_{23} \mathrm{NO}_{4} \mathrm{H} \mathrm{Br}+4 \mathrm{H}_{2} \mathrm{O}$

$\mathrm{H}_{2} \mathrm{O}=13,89$ Proz.

\begin{tabular}{cc}
\multicolumn{3}{c}{ Gefunden : } \\
II. & IV. \\
17,98 & 17,85 \\
\multicolumn{2}{c}{ Gefunden : } \\
I. & III. \\
14,13 & 15,02
\end{tabular}


Jodwasserstoffsaures Dehydrocorydalin.

Beim Erwärmen der Acetonverbindung mit verdünnter Jodwasserstoffsäure scheidet sich ein amorpher, gelber Niederschlag aus, der, in heifsem Alkohol gelöst, nach dem Erkalten dieselben charakteristischen, kleinen Nadeln liefert, welche durch Einwirkung von Jod auf Corydalin gewonnen werden.

Saures sehwefelsaures Dehydrocorydalin.

$$
\mathrm{C}_{22} \mathrm{H}_{23} \mathrm{NO}_{4}, \mathrm{H}_{2} \mathrm{SO}_{4}+3 \mathrm{H}_{2} \mathrm{O} \text {. }
$$

Behandelt man die Acetonverbindung in analoger Weise mit verdünnter Schwefelsäure, so erhält man eine wässrige Lösung des sauren Salzes, aus der nach dem Erkalten prismatische, gelbe Krystalle anschiefsen. Dieselben sind durch Unkrystallisation aus warmem Wasser zu reinigen.

I. 0,208 $\mathrm{g}$ Substanz verloren bei $10000,0217 \mathrm{~g}=10,43$ Proz. $\mathrm{H}_{2} \mathrm{O}$.

II. $0,2209 \mathrm{~g}$ Substanz verloren $0,0228 \mathrm{~g}=10,27$ Proz. H.

III. $0,2226 \mathrm{~g} \quad, \quad 0,023 \mathrm{~g}=10,33$, ,

IV. Bei der Schwefelsäurebestimmung liøferten $0,1863 \mathbf{g}$ getrocknete Substanz 0,0935 g Ba SO $4=21,15$ Proz. $\mathrm{H}_{2} \mathrm{SO}_{4}$.

V. Bei der Elementaranalyse im beiderseits of Gemisch von gekörntem Kupferoxyd und Bleichromat beschickten Rohre mit vorgelegter reduzierter Kupferspirale liefurten 0,1981 g getrocknete Substanz 0,4134 g $\mathrm{CO}_{3}$ und $0.0984 \mathrm{H}_{2} \mathrm{O}$.

VI. Eine Elementaranalyse in einem ebenso gefüllten Rohr ergab aus $0,1996 \mathrm{~g}$ getrockneten Salzes $0,4158 \mathrm{~g} \mathrm{CO}_{2}$ und $0,0986 \mathrm{~g} \mathrm{H}_{2} \mathrm{O}$.

$$
\begin{array}{rlcccccc}
\text { Ber.: } & \multicolumn{1}{c}{\text { Gef.: }} & \text { I. } & \text { II. } & \text { III. } & \text { IV. } & \text { V. } & \text { VI. } \\
\mathrm{H}_{2} \mathrm{O} & =10,44 & 10,43 & 10,27 & 10,33 & - & - & - \\
\mathrm{H}_{2} \mathrm{SO}_{4} & =21,16 & - & - & - & 21,15 & - & - \\
\mathrm{C} & =57,02 & - & - & - & - & 56,91 & 56,81 \\
\mathrm{H} & =5,39 & \cdots & - & - & - & 5,50 & 5,46
\end{array}
$$

Der gefundene Wassergehalt dentet auf eine Formel mit 3 Molekülen Krystallwasser hin. Die gefundenen Werte von $\mathrm{C}$ und $\mathrm{H}$ berechtigen zu der Annahme, dals ein Salz mit 23 Atomen $\mathrm{H}$ vorliegt. Denn das schwefelsaure Salz von der Formel $\mathrm{C}_{22} \mathrm{H}_{25} \mathrm{NO}_{4}, \mathrm{H}_{2} \mathrm{SO}_{4}$ würde

$$
\begin{array}{rr}
56,77 \text { Proz. } & \text { C. } \\
5,80 \quad \text { H. }
\end{array}
$$

enthalten. Die gefundenen Daten bestätigen daher die frühere Annahme, dafs dem Corydalin die Formel $\mathrm{C}_{22} \mathrm{H}_{27} \mathrm{NO}_{4}$ und dem Dehydrocorydalin $\mathrm{C}_{22} \mathrm{H}_{23} \mathrm{NO}_{4}$ zuzuerteilen ist. 
Salpetersaures Dehydrocorydalin.

$$
\mathrm{C}_{23} \mathrm{H}_{23} \mathrm{NO}_{4} \mathrm{HNO}_{3}+2 \mathrm{H}_{2} \mathrm{O} \text {. }
$$

Nur bei einer Probe ist es mir gelungen, das Nitrat direkt aus der Acetonverbindung zu gewinnen, indem ich zu der erwärmten Mischung derselben mit Wasser tropfenweise verdünnte Salpetersäure zufügte, bis schwach saure Reaktion und zugleich Lösung eingetreten war. Es krystallisierten daraus dunkelgelb gefärbte Nadeln. Nach dem Eindampfen der Mutterlaugen schieden sich nur noch braune, amorphe Massen ab: jedenfalls nur Oxydationsprodukte. Auch wenn die Acetonverbindung in starkem Alkohol durch ganz gelindes Erwärmen gelöst und tropfenweise verdünnte Salpetersäure bis zur sauren Reaktion zugesetzt wurde, resultierten nur braune, die Oberflache bedeckende und an den Wandungen des Gefälses haftende Schuppen. Hingegen konnte ich aus den Filtraten von den Halogenbestimmungen der Delydrocorydalinsalze das salpetersauro Salz leicht isolieren. Namentlich boi der Analyse der Chloride und Bromide, die in Wasser leicht löslich sind, konnte ich durch $\mathbf{Z u}$ fügen von einigen Tropfen Salpetersäure und von Silbernitrat im geringen Ueberschufs nach dem Abfiltrieren von dem ausgeschiedenen Halogensilber Lösungen des Nitrats erhalten, aus denen es sich nach dem Erkalten und weiter beim Verdunsten im Exsiccator in gelben, verhältnismäfsig langen Nadeln ausschied. Aus Wasser umkrystallisiert, zeigten die angeschossenen, gelben Krystalle gleichfalls die Form von Nadeln.

$0,2030 \mathrm{~g}$ lufttrockener Substanz verloren bis zum konstanten Gewicht bei $100^{\circ}$ getrocknet $0,0162 \mathrm{~g} \mathrm{H}_{2} \mathrm{O}=7,98$ Proz. $\mathrm{B}_{2} \mathrm{O}$.

$0,1868 \mathrm{~g}$ derartig getrockneter Substanz lieferten bei der Verbrennung im Kupferoxyd-Bleichromatrohr mit vorgelegter reduzierter Kupferspirale $0,4203 \mathrm{~g} \mathrm{CO}_{2}$ und $0,0956 \mathrm{~g} \mathrm{H}_{2} \mathrm{O}$.

Berechnet sind für $\mathrm{C}_{22} \mathrm{H}_{23} \mathrm{NO}_{4} \mathrm{H} \mathrm{NO}_{3}+2 \mathrm{H}_{2} \mathrm{O}$

$$
\mathrm{H}_{2} \mathrm{O}=7,75 \text { Proz. }
$$

Gefunden $\mathrm{H}_{2} \mathrm{O}=7,98$ Proz.

Berechnet fur $\mathrm{C}_{22} \mathrm{H}_{23} \mathrm{NO}_{4}, \mathrm{H} \mathrm{NO}$

Gefundon

$$
\begin{array}{ll}
\mathrm{C}=61,68 \text { Proz. } & \mathrm{C}=61,34 \text { P.oz. } \\
\mathrm{H}=5,60, & \mathrm{H}=5,67 .
\end{array}
$$


Debydrocorydalingoldchloridausder Aceton-

$$
\begin{gathered}
\text { ve r b ind un g. } \\
\mathrm{C}_{22} \mathrm{H}_{23} \mathrm{NO}_{4} \mathrm{H} \mathrm{Cl} \mathrm{Au} \mathrm{Cl} \\
\text {. }
\end{gathered}
$$

Das Golddoppeisalz stellte ich ebenso, wie oben dar, indem ich eine aus der Acetonverbindung gewonnene, wässerige Lösung des salzsauren Dehydrocorydalins mit Goldchloridlösung versetzte, den braunroten Niederschlag absaugte, auswusch und aus salzsäurehaltigem, absoluten Alkohol umkrystallisierte. Ich erhielt die gleichen braunroten, charakteristischen kleinen Nadeln vom Schmelzpunkt $219^{\circ}$.

0,2137 g Substanz hinterlielsen

$$
\begin{aligned}
& 0,0597 \mathrm{~g} \mathrm{Au}=27,94 \text { Proz, Au. } \\
& \text { Berechnet 27,91 } \text { (Au. }
\end{aligned}
$$

Dehydrocorydalinplatinchlorid a us der Acetonverbindung.

$$
\left(\mathrm{C}_{22} \mathrm{H}_{23} \mathrm{NO}_{4} \cdot \mathrm{H} \mathrm{Cl}\right)_{2} \mathrm{Pt} \mathrm{Cl} \mathrm{Cl}_{4}+6 \mathrm{H}_{2} \mathrm{O} \text {. }
$$

Die Darstellung gesehah in ganz enteprechender Woise, wio bei dem früber erbaltenen Dehydrocorydalinplatinchlorid. Die ans Alkohol umkrystallisierten gelben Nadeln ergaben folgende Werte:

$0,2086 \mathrm{~g}$ luftrockener Substanz bei $100-105^{0}$ getrocknet gaben ab $0,0182 \mathrm{~g} \mathrm{H}_{2} \mathrm{O}=8,72$ Proz. $\mathrm{H}_{2} \mathrm{O}$.

0,1904 getrocknetes Salz hinterliefs $0,0332 \mathrm{~g} \mathrm{Pt}=17,43$ Proz. Pt.

Berechnet:

$$
\begin{aligned}
\mathrm{H}_{2} \mathrm{O} & =8,61 \\
\mathrm{Pt} & =17,21
\end{aligned}
$$

Gefunden :

$8, \pi 2$

17,43

Chloroform-Dehydrocorydalin.

$$
\mathrm{C}_{22} \mathrm{H}_{23} \mathrm{NO}_{4} \cdot \mathrm{CHCl}_{3} \text {. }
$$

E. S c h m dit ${ }^{1}$ hat zuerst aus dem Berberin ein Chloroform.Berberin $\mathrm{C}_{20} \mathrm{H}_{17} \mathrm{NO}_{4} . \mathrm{CHCl}_{3}$ als gut krystallisierende, sehr beständige Verbindung dargestellt. Die sonstigen, mit dem Berberin übereinstimmenden Reaktionen des Dehydrocorydalins liefsen vermuten, dafs dasselbe anch eine ähnliche, charakteristisch $\theta$ Verbindung geben wïrde. Nach E. $\mathrm{Schmidt}$ wurde $1,0 \mathrm{~g}$ fein zerriebenes Dehydrocorydalinhydrojodid mit $50 \mathrm{ccm}$ Wasser angeschüttelt, die Mischung mit $30 \mathrm{ccm}$ Chloroform ver-

1) Arch. d. Pharm. 1897. 
setzt und mit Natronlauge stark alkalisch gemacht. Die Flüssigkeit wurde einige Zeit auf dem Dampf bade erwärmt und wiederholt kräftig. durchgeschüttelt. Dabei wurde die vorher gelb gefärbte wässerige Schicht fast farblos, wäbrend das Chloroform gelblich gefärbt erschien. Im Scheidetrichter wurde das Chloroform von der alkalischen Flüssigkeit getrennt und wiederholt mit Wasser ausgeschüttelt. Darauf wurde der grölste Teil des Chloroforms abdestilliert und der Rest der freiwilligen Verdunstung überlassen. Es blieb ein brauner Rückstand, der, nach dem Abspülen mit Alkohol, in wenig warmem Chloroform gelöst wurde, worauf die klare, braune Flüssigkeit in einem verschliếsbaren Gefäfs mit viel Alkohol überschichtet wurde. Nach längerem Stehen schieden sich tafeltörmige, fast tarblose Krystalle ab, ähnlich denen, welche das Chloroform-Berberin darstellte. Der Schmelzpunkt der zwischen Flielspapier getrockneten Krystalle lag bei $162-1630$. Bei $100^{\circ}$ erlitt die zerriebene Substanz k e i n e n Gewichtsverlust.

0,2327 g Substanz lieferten nach Carius 0,2073 g Ag Cl $=$ 22,03 Proz. Cl.

Berechnet für $\mathrm{C}_{22} \mathrm{H}_{23} \mathrm{NO}_{4} \cdot \mathrm{CHCl}_{3}: \mathrm{Cl}=21,98$ Proz.

Einwirkung von Schwefelammonium auf Dehydrocorydalinhydrojodid.

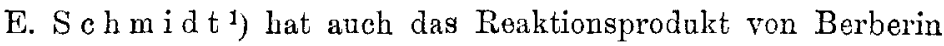
und gelbem Schwefelammonium zuerst dargestellt, und $\mathrm{G} \mathrm{a} \mathrm{z}^{2}{ }^{2}$ ) hat alsdann weitere Untersuchungen hierüber angestellt. Iss schien von Interesse zu sein, auch das Debydrocorydalin nach dieser Richtung hin oiner Prüfung za unterziohen.

$\mathrm{Zu}$ einer verdünnt-alkoholischen heifsen L ös u n g des jodwasserstoffsauren Salzez wurde daher gelbes Schwefolanmonium hinzugefügt. Sehr bald, namentlich nach dem Erkalten, sclieden sich kleine, braune Nadeln ab, die in dem Verbalten eine grolse Aehnlichkeit mit dom Berberinwasserstoffpolysulfid zeigten.

Auch die Uebereinstimmung dieser Reaktion mit der des Berberins beweist wieder die nahe Verwandtschaft der beiden Alkaloide.

1) Arch. d. Pharm. 1887, p. 148.

2) $» " 1890$, p. 631 . 
Reduktion des Dehydrocorydalins.

Um zu konstatieren, ob das Dehydrocorydalin, entsprechend dem Berberin, bei der Reduktion wieder vier Atome Wasserstoff, ev. unter Rückbildung von Corydalin, autnimmt, suspendierte ich $5,0 \mathrm{~g}$ Aceton-Dehydrocorydalin in Wasser, fügte verdünnte Schwefelвäure im grofsen Ueberschuls und Zinkpulver hinzu und vermehrte die Wasserstoffentwicklung durch zeitweiliges gelindes Erwärmen auf dem Wasserbade. Nach einigen Tagen war die anfangs intensiv gelb gefärbte Flüssigkeit fast farblos geworden. Ich filtrierte nun die Lösung vom uberschüssigen Zink ab, fügte so viel starke Natronlauge zu, bis der anfangs ausgeschiedene Brei von Zinkbydroxyd in Lösung gegangen war, trennte durch Filtrieren den weilsen Niederscblag von der Flülsigkeit und schüttelte ihn noch feucht wiederholt mit Aether aus. Die ätherische, schwach gelb getärbte Lösung engte ich durch Abdestillieren auf ein kleines Volumen ein. Nach einiger Zeit schieden sich aus der dunkel gefärbten Flüssigkeit farblose Krystalle aus, die aus Alkohol umkrystallisiert wurden. Sie zeigten eine ähnliche Form wie das Corydalin und den Schmelzpunkt desselben $135^{\circ}$. Sie ergaben auch, wie aus der beigefügten Tabelle zu ersehen ist, dieselben Reaktionen wie das Corydalin und färbten sich gleichfalls am Licht gelb. Auch eine Elementaranalyse bestätigte, dafs durch die Reduktion des Dehydrocorydalins wieder vier Atome $\mathrm{H}$ in das Molekül eingetreten sind.

$0,2400 \mathrm{~g}$ Substanz im Exsiccator getrocknet, lieferten im Bleichromat-Kupferoxydrohr $0,6266 \mathrm{~g} \mathrm{CO}_{2}$ und $0,1524 \mathrm{~g} \mathrm{H}_{2} \mathrm{O}$.
Berechnet für:
Gefunden :
$\mathrm{C}_{22} \mathrm{H}_{27} \mathrm{NO}_{4}$
$\mathrm{C}=71,54$ Proz.
71,20 Proz.
$\mathbf{H}=7,32$
7,5

Es lag daher die Vermutung nahe, dafs durch die Reduktion des Dehydrocorydalins das Corydalin mit allen seinen Eigenschaften wieder gewonnen wäre. Zur Bestätigung dieser Annahme versuchte ich, das Goldsalz darzustellen, welches bei dem naturellen Corydalin aufserordentlich charakteristisch ist.

Hierbei machte ich jedoch Wahrnehmungen, die nur auf ein Vorhandensein einer mit dem Corydalin isomeren Base schliefsen liefsen. 
Methoxylbestimmungen im Dehydrocorydalinbydrojodid.

Dobbie und La u d e ${ }^{1}$ ) haben im Corydalin vier Methoxyle nachgewiesen. Demnach war es wahrscheinlich, dals auch in dem Dehydrocorydalin gleichfalls $4\left(\mathrm{O} . \mathrm{CH}_{3}\right)$ vorhanden wären. Zum Nachweis hiervon verfuhr ich in der von $Z$ eise ${ }^{2}$ ) ange. gebenen Methode, nach welcher durch Einwirkung starker Jodwasserstoffsäur $\theta$ vom Siedepunkt $127^{\circ}$ aus den Methoxylgruppen die

Methyle in Form von Jodmethyl abgespalten werden, welches. dann in alkoholischer Silbernitratlösung Jodsilber abscheidet.

$0,2397 \mathrm{~g}$ lufttrockenes jodwasserstoffsaures Dehydrocorydalin lieferte bei diesor Bestimmung $0,4297 \mathrm{~g} \mathrm{Ag} \mathrm{J}=23,65$ Proz, $\mathrm{O} \cdot \mathrm{CH}_{3}$. Berechnet sind für die Formel $\mathrm{C}_{22} \mathrm{H}_{23} \mathrm{NO}_{4} \mathrm{HJ}+2 \mathrm{H}_{2} \mathrm{O}$ für $4\left(\mathrm{O} \cdot \mathrm{CH}_{3}\right)=23,44$ Proz.

Es dürfte hierdurch der Beweis erbracht sein, dafs auch das: Dehydrocorydalin vier Methoxylgruppen enthält.

Ich versuchte nun, diesen entmethylierten Körper rein darzastellen, weil eine Analyse desselben in reiner Gestalt mit noch gröfserer Genauigkeit zwischen der wahrscheinlicheren und unwahrscheinlicheren Formel des Corydalins entscheiden mulste.

Aus der nach Abspaltung der vier Methylgruppen resultierenden braunen Flüssigkeit schieden sich beim Erkalten gelbbraune Krystalle ab. Nach Entfernung der überschüssigen Jodwasserstoffsäure lösten sich dieselben in heifsem Alkohol auf, ohne jedoch wieder ans demselben zu krystallisieren. Auch nach anhaltendem Kochen mit Chlorsilber und Salzsäure krystallisierte aus der filtrierten Flülsigkeit Nichts aus. Beim Zufügen von Gold- und Platinchlorid zu kleinen Proben trat sofort Reduktion ein. Auch der Versuch, mit Silbernitrat die ganze Menge in das Nitrat umzusetzen, führte zu keinem Ziele.

Es wurde daher eine neve Menge jodwasserstoffsauren Dehydrocorydalins mit der fünffachen Menge starker Jodwasserstoffsäure obne Zusatz von amorphem Phosphor auf der Asbestpappe ca. zwei Stunden lang am Steigrohr erhitzt, die überschüssige Säure in eine Schale abgegossen und abgedampft, die im Kolben abgeschiedene

1) Chem. Pharm. Curbi. 1892. II. p. 220.

2) Monatshefte f. Chem, 18ธ5. pag. 959. 
Krystallmasse mit verdünnter Jodwasserstoffsäure in eine andere Schale hineingespült. Nach langem Erhitzen auf dem Dampfbade hatten sich in der Mitte der Schale krystallinische, hellgelbeKörper angesetzt, die von einen braunen Rand umgeben waren. Die ganze Menge wurde nochmals mit verdünnter Jodwasserstoffsäure durchfeuchtet und von Neuem zur Trockne verdampft. Der braune Rand war kleiner, die krystallinischen, gelben Körper heller geworden, Ich brachte dieselben nun in ein kleines Becherglas und spülte sie mebrere Male mit starkem kalten Alkohol, in dem sie sich als schwer löslich erwiesen, ab und trocknete sie schliefslich aut dem Damptbade. In dieser Form verwendete ich sie zu den Analysen.

I. 0,2938 g Substanz ergaben nach Carius 0,1575 g Ag J $=$ 28,97 Proz. J.

II. Bei der Verbrennung im Bleichromatrohr mit vorgelegter reduzierter Kupferspirale lieferten $0,2477 \mathrm{~g}$ Substanz $0,4448 \mathrm{~g} \mathrm{CO}_{2}$ und $0,0989 \mathrm{~g} \mathrm{H}_{2} \mathrm{O}$.

III. Eine andere, aber nach derselben Weise dargestellte Probe lieferte von $0,2464 \mathrm{~g}$ getrockneter Substanz $0,4446 \quad \mathrm{~g} \quad \mathrm{CO}_{2}$ und $0,0927 \mathrm{~g} \mathrm{H}_{2} \mathrm{O}$.

\begin{tabular}{ccccc} 
Berechnet für: & \multicolumn{3}{c}{ Gefunden: } & Berechnet fur : \\
$\mathrm{C}_{18} \mathrm{H}_{15} \mathrm{NO}_{4} \mathrm{HJ}$ & $\mathrm{I}$. & II. & III. & $\mathrm{C}_{18} \mathrm{H}_{17} \mathrm{NO}_{4} \mathrm{HJ}$ \\
$\mathrm{J}=29,06$ & 28,97 & - & - & $\mathrm{J}=28,96$ \\
$\mathrm{C}=49,42$ & - & 48,98 & 49,16 & $\mathrm{C}=49,20$ \\
$\mathrm{H}=3,66$ & - & 4,40 & 418 & $\mathrm{H}=4,10$
\end{tabular}

Obschon die gefundenen Werte besser mit der Formel $\mathrm{C}_{18} \mathrm{H}_{17} \mathrm{NO}_{4} \mathrm{HJ}$, welche weiter auf $\mathrm{C}_{23} \mathrm{H}_{29} \mathrm{NO}_{4}$ als richtige Formel des Corydalins hinweisen würde, im Einklange stehen, so stehen sie doch auch nicht mit der Formel $\mathrm{C}_{18} \mathrm{H}_{15} \mathrm{NO}_{4} \mathrm{HJ}$ im Widerspruch, namentlich wenn man erwägt, dafs die fragliche Verbindung nicht umkrystallisiert werden konnte.

Nach den bisher vorliegenden Beobachtungen scheint mir die Formel $\mathrm{C}_{22} \mathrm{H}_{27} \mathrm{NO}_{4}$ für das Corydalin, unter Berücksichtigung der fast übereinstimmenden Werte, die Dobbie und L a uder, Fre und und $J$ oseph $i$ und ich bei der Analyse der Base gofundenhaben, die richtigere zu sein. Es sprechen dafür ferner die von mir bei der wiederholten Analyse des salpetersauren Corydalins ormittelten Daten, und weiter die Worte, welche dia Analyso des sauren schwefelsauren Dehydrocorydalins ergab. 
Ferner scheint es mir durch die vorstehenden Versuche nachgewiessn zu sein, dafs eine direkte Beziehung des Corydalins zum Berberin besteht.

Dem Berberin kommt die Formel $\mathrm{C}_{20} \mathrm{H}_{17} \mathrm{NO}_{4} \mathrm{zu}$, es unterscheidet sich also von dem Dehydrocorydalin $\mathrm{C}_{22} \mathrm{H}_{23} \mathrm{NO}_{4}$ durch einen Mindergehalt von $2 \mathrm{CH}_{3}$. Berücksichtigt man, dafs das Berberin 2 Methoxylgruppen und wahrscheinlich eine Oxymethylengruppe enthält, das Dehydrocorydalin hingegen 4 Methoxylgruppen, so könnte man vermuten, dafs die Formeln beider Basen zu schreiben seien:

$$
\begin{gathered}
\mathrm{C}_{17} \mathrm{H}_{9} \mathrm{~N}\left(\mathrm{O} . \mathrm{CH}_{3}\right)_{2}{ }_{\mathrm{O}}^{\mathrm{O}}>\mathrm{CH}_{2}=\text { Berberin. } \\
\mathrm{C}_{18} \mathrm{H}_{11} \mathrm{~N}\left(\mathrm{O} . \mathrm{CH}_{3}\right)_{4} \text { Dehydrocorydalin. }
\end{gathered}
$$

Weitere Untersuchungen werden lehren, ob diese naheliegenden Beziehungen der beiden Bagen die richtigen sind.

Goldsalzdes reduzierten Dehydrocorydaling.

$$
\mathrm{C}_{22} \mathrm{H}_{27} \mathrm{NO}_{4} \cdot \mathrm{H} \mathrm{Cl}, \mathrm{Au} \mathrm{Cl} 3+4 \mathrm{H}_{2} \mathrm{O} \text {. }
$$

Stellte ich unter ganz den gleichen Bedingungen wie beim Corydalin aus dem reduzierten Dehydrocorydalin durch Fällen mit Goldchlorid in wässeriger Lösung das Goldsalz dar, saugte den gelblich weifsen Niederschlag ab und krystallisierte ihn aus Alkohol um, so zeigte schon die alkoholische Lösung nicht die gelbrote, sondern eine rein hellgelbe Farbe. Noch auffalliger war der Unterschied in der Färbung, Form und Löslichkeit der Krystalle. Während das Corydalingoldchlorid rote, büschelig angeordnete, oft ziemlich derbe, in Alkohol schwer lösliche Nadeln bildete, waren die Krystalle des Goldsalzes des reduzierten Dehydrocorydalins hellgelbe, teine, sehr schön ausgebildete, leicht lösliche Säulen. Auch die Analyse bestätigte, dals ein ganz anderes Goldsalz vorlag und zwar ein normales von der Formel $\mathrm{C}_{2 a} \mathrm{H}_{27} \mathrm{NO}_{4} \cdot \mathrm{H} \mathrm{Cl} . \mathrm{Au} \mathrm{Cl} 3+4 \mathrm{H}_{2} \mathrm{O}$.

Bei längerem Liegen an der Luft verlor das Salz einen Teil des Krystallwassers, woher es kommen mag, dafs nur bei einem frisch dargestellten und sofort analysierten Präparat der berechnete Krystallwassergehalt fast vollständig gefunden wurde.

Beim Trocknen bei $100^{\circ}$ nahm das Goldsalz eine braune Färbung an. 


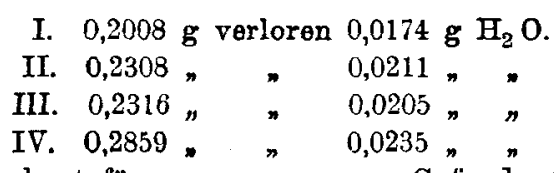

Berechnet für: 4 Moleküle $\mathrm{H}_{2} \mathrm{O}$ $\mathrm{H}_{2} \mathrm{O}=9,22$ Proz.

I. II. III.

$8,66 \quad 9,14 \quad 8,85 \quad 8,22$.

V. $0,1834 \mathrm{~g}$ getrocknete Substanz hinterlielsen $0,0511 \mathrm{~g}$ Au.

VI. 0,2097 g getrocknete Substanz 0,0583 g Au.

VII. Bei der Elementaranalyse im Bleichromatrohr fand ich aus $0,2111 \mathrm{~g}$ getrockneter Substanz $0,2884 \mathrm{~g} \mathrm{CO}_{2}$ und $0,0742 \mathrm{~g} \mathrm{H}_{2} \mathrm{O}$.

Bei den Chlorbestimmungen machte ich dieselbe Erfahrung wio beim Corydalingoldchlorid. Ich führte daher eine Analyse ähnlich wie dort durch Kochen der getrockneten Substanz mit konzentrierter Natriumkarbonatlösung aus und fand dabei aus

VIII. $0,2624 \mathbf{g}$ getrockneter Substanz 0,2114 $\mathbf{g ~ A g ~ C l . ~}$

\section{Gefunden:}

V. VI. VII. VIII.

$\begin{array}{lccc} & \text { V. } & \text { VI. } & \text { VII. } \\ \mathrm{Au} & 27,86 & 27,80 & - \\ \mathrm{C} & - & - & 37,23 \\ \mathrm{H} & - & - & 3,90 \\ \mathrm{Cl} & - & - & - \\ & \text { Berechnot } & \text { für } \mathrm{C}_{22} \mathrm{H}_{27} \mathrm{NO}_{4} . \mathrm{H} \\ & & \mathrm{Au}=27,75 & \text { Proz. } \\ \mathrm{C} & =37,25 \\ \mathrm{H} & =3,95, \\ & \mathrm{Cl}=20,03\end{array}$

Die weiteren Untersuchungen der durch Reduktion des Dehydrocorydalins erhaltene Base werden lehren, ob dieselbe auch in anderer Beziehung Verschiedenheiten von dem naturellen Corydalin zeigte.

Balboc apnin.

$$
\mathrm{C}_{19} \mathrm{H}_{19} \mathrm{NO}_{4} \text {. }
$$

A d e rman $n^{1}$ ) hat auch dieses Alkaloid dargestellt und 83 mit „Corydalin“ bezeichnet. Er giebt an, dafs sich seine Base B mit grau-grüner Farbe in Aetzalkalien löst, ein Verhalten, welches von allen Corydalisalkaloiden nur das Bulbocapnin zeigt. Das salzsaure Salz krystallisiert nach A d er ma n n's Mitteilungen aus verdünnten Lösungen sehr leicht in feinen, seidenglänzenden, langen Nadeln aus konzentrierten, dagegen körnig krystallinisch. Auch dieses Ver-

1) Inaug. Dissert. Dorpat 1890. 
halten ist charakteristisch für das Bulbocapnin. Adermann glaubt dieser Base die Formel $\mathrm{C}_{22} \mathrm{H}_{22} \mathrm{NO}_{4}$ zuerteilon zu sollen.

F reund und $\mathrm{J}$ oseph $\mathrm{i}^{1}$ ) haben den Namen Bulbocapnin für die bei $199^{\circ}$ schmelzende Corydalisbase eingeführt.

Dieselbe krystallisiert nach Angaben dieser Forscher aus Alkohol in Rhomben und löst sich leicht in Chloroform. Vom Corydalin unterscheidet sich das Bulbocapnin besonders dadurch, dals es in Aetzalkalien mit grüner Farbe löslich ist, eine Färbung, welche auch die konzentrierten Salzlösungen zeigen. Es ist nicht so lichtempfindlich wie das Corydalin, wenigstens kann das salzsaure Salz tagelang selbst im direkten Sonnenlichte stehen, ohne seine Farbe zu verändern. Das salzsaure Bulbocapnin krystallisiert im Gegensatz zu dem salzsauren Corydalin sehr leicht, und zwar in seidenglänzenden, langen, zu Rosetten angeordneten Nadeln; aus konzentrierter Lösung jedoch in körnig-krystallinischer Gestalt.

Fre und und Jose $\mathrm{phi}^{2}$ ) haben dem Bulbocapnin zuerst die Formel $\mathrm{C}_{34} \mathrm{H}_{3} \mathrm{~N}_{2} \mathrm{O}_{7}$ beigelegt, dieselbe jedoch später ${ }^{3}$ ) in $\mathrm{C}_{19} \mathrm{H}_{19} \mathrm{NO}_{4}$ umgeändert.

Die von mir mit selbstdargestellten Bulbocapnin vom Schmp. $199^{\circ} \mathrm{im}$ Kupforoxydrohre ausgeführte Elementaranalyse ergab folgende Werte:

\section{$0,2416 \mathrm{~g}$ Subst. lieferten $0,6204 \mathrm{~g} \mathrm{CO}_{2}$ und $0,1314 \mathrm{~g} \mathrm{H}_{2} \mathrm{O}$.}

Bei der Stickstoffbestimmung nach Will und Varrentrapp brauchten $0,2336 \mathrm{~g}$ Subst. $7.90 \mathrm{ccm} 1 / 10 \mathrm{~N} . \mathrm{HCl}=4,73$ Proz. N.

$\begin{array}{cc}\text { Berechnet für : } & \text { Gefunden } \\ \mathrm{C}_{19} \mathrm{H}_{19} \mathrm{NO}_{4} & \\ \mathrm{H}=7015 & 70,03 \\ \mathrm{H}=5,84 & 6,04 \\ \mathrm{~N}=4,30 & 4,73\end{array}$

Die gefundenen Werte stimmen mit der von Freund und Jose phi aufgestellten Formel $\mathrm{C}_{19} \mathrm{H}_{19} \mathrm{NO}_{4}$ überein. Ich habe daher keine weiteren Analysen ausgeführt, auch keine Salze dargestellt, condern nur das Verhalten des Bulbocapnins gegen Jod studiert, und zwar habe ich zu diesen Versuchen ein von E. M erck bezogenes Bulbocapnin vom Schmp. $199^{\circ}$ verwendet.

1) Annal. d. Chem, 277. p. 10 .

2) Ber. d. deutschen chem. Ges. 25. 2413.

3) Arnal. d. Chemie 277. p. 10, 
Verhalten des Bulbocapnins gegen Jod.

Beim tropfenweisen Zufügen von alkoholischer Jodlösung $\mathrm{zu}$ alkoholischer Bulbocapninlösung ging die braun Farbe nicht wie bei dem Corydalin in eine gelbe über, vielmehr zeigte sich anfangs eine dunkelgrüne Färbung, die bei weiterem Jodzusatz schwarzgrün wurde. Darauf wurden $0,4355 \mathrm{~g}$ Bulbocapnin in alkoholischer Lösung mit 2,1205 g getrockneten Jods in einer Druckflasche drei Stunden im Dampf bade erwärmt. Nach dem Erkalten hatten sich am Boden und an den Wandungen der Flasche schwarze, kohleartige Massea ausgeschieden, während die Flüssigkeit selbst braunschwarz getärbt. war. Ich fügte nun $180 \mathrm{ccm} 1 / 10 \mathrm{~N} . \mathrm{Na}_{2} \mathrm{~S}_{2} \mathrm{O}_{3}$ Lösung, d. h. einen geringen Ueberschuls derselben, zu, ferner wenig Natriumbicarbonat und etwa $2,0 \mathrm{~g}$ Jodkajium, und erwärmte die Miscling mehrere Tage lang auf dem Wasserbade. Währond beim Corydalin sohr bald eine Zerlegung der gebildeten Perjodide eintrat, blieb hier die Lösung grau-schwarz gefärbt, und die schwarzen Partikeln veründerten sich auch dann nicht, als ich unter Anfgabe des quantitativen Versuchs, zu einer Probe wässrige schwellige Säure binzufügte. Weiter angeatellte Versuche, durch Kalilauge die Perjodide zu zerlegen und die freigemachte Base durch Aether anszuschütteln, führten zn keinem Resultate. Iob dampfte nun die schwarze Flüssigkeit ein, filtrierte die ausgeschiedenen, kohleartigen Massen ab und wusch sie mit Wasser sorgfällig aus. Eine Probe derselben schüttelte ich mit Wasser an und versuchte sie durch Erhitzen mit feuchtem Chlorsilber umznsetzen ; es trat jedoch keine merkliche Veränderung der wässrigen Flüssigkeit ein, auch die schwarzen Partikeln blieben zusammengeballt. Erst nach dem Hinzufügen einiger Tropfon Salzsüure und nach mehrmaligem Aufkochen, wurde die Flüssigkeit tiefschwarz, indem die dunklen Massen in Lösung gingen. Nach dem Erkalten filtrierte ich das überschüssige $\mathrm{Ag} \mathrm{Cl}$ und gebildete $\mathrm{Ag} \mathrm{J}$ $a b$, versetzte die Flüssigkeit mit einigen Tropten Bleiessig und leitete $\mathrm{H}_{2} \mathrm{~S}$ ein. Das ausgeschiedene $\mathrm{PbS}$ entfernte ich durch $\mathrm{Ab}$ filtrieren, den Schwefelwasserstoff durch Eindampten und liefs einige Tropfen der nun heller gefärbten Flüssigkeit auf einem Uhrglase. freiwillig verdunsten : neben noch grauschwarzen, nicht krystallisierten Massen, zeigten sich einige farblose, tafelförmige Krystalle. Auch 
gab die Lösung mit Grold- und Platinchlorid gut ausgebildete, nadelförmige Krystalle, während vor der Behandlung mit Bleiessig nur -schwarze, amorphe Fällungen eingetreten waren.

Nach diesem, mit einer kleinen Probe angestellten Versuch, behandelte ich die Menge der kohligen Massen in gleicher Weise, konnte jedoch, auch nach wiederholtem Zusatz von Bleiessig und Entferuen des Bleies mit Schwefelwasserstoff, keine ungetärbta Lösung und nach dem Verdunsten nur wenig farblose Krystalle orhalten.

Ich gab daher diesen Versuch auf, weil durch jene Beobachtungen bereits dargethan war, dafs bei der Einwirkung von Jod auf Bulbocapnin kein gelber, dem Berberin verwandter Körper gobildet wird.

Bulbocapnin und Jodmethyl in alkalischer L ös ung.

Fround und Jose phi $\mathrm{i}^{1}$ ) haben durch Digestion von Bulbocapnin mit überzchüssigem Jodmethyl das Jodmethylat

$$
\mathrm{C}_{19} \mathrm{H}_{19} \mathrm{NO}_{4} \cdot \mathrm{CH}_{3} \mathrm{~J}
$$

in bei 25:0 schmelzenden glänzenden Tafeln erhalten. Die beiden Forscher geben an, dars in dem Bulbocapnin eino Mothoxylgruppe und wahrscheinlich drei Hydroxylgruppen ${ }^{2}$ ) vorhanden sind. Die drei H Atome der Hydroxyle versuchte ich durch Methyl zu orsetzen, um dadurch einen Körper zu erhalten, dem die Formel $\mathrm{C}_{22} \mathrm{H}_{25} \mathrm{NO}_{4}$ zukommen würde.

$\mathrm{Zu}$ diesem Zwecke löste ich $1,0 \mathrm{~g}$ Bulbocapnin in Methylalkohol auf, fügte der Flüssigkeit eine Lösung der berechneten Menge KOH (3 Moleküle $\mathrm{KOH}$ aut ein Mol. Bulbocapnin) $=0,0 \mathrm{~g}$ in Methylalkohol, sowie überschüssiges Jodmethyl zu und erhitzte die Mischung unehrere Stunden lang in einer Druckflasche im Wasserbade. Die in der Kälte grüne Flüssigkeit, herrührend von der Lösung des Bulbocapvins in überschüssigem Alkali, zeigte nach der Einwirkung eine gelbbraune Farbe. Beim Verdunsten des Methylalkohols und des überschüssigen Jodmethyls auf dem Wasserbade blieben grüuliche Krystalle zurück, von denen ich eine Probe in

1) Anm. d. Chem. 277. p. 14.

2) " " 27. p. 15 
schwach salzsäurehaltigem Wasser durch Kochen auflöste. Die erhaltene klare Flüssiglreit teilte ich in zwei Teile und fügte zu dem einen wach dem Erkalten verdünnte Natronlauge, zu dem anderen Natriumcarbonatlösung zu: es trat jedoch bei boidon zunächst keine Fällung ein, ein Beweis dafür, dafs unverändertes. Bulbocapnin nicht mehr vorhanden sein konnte. Erst nach mehrstündigem Stehen schieden sich aus der mit Natronlauge alkalisch gemachten Probe durchsichtige Krystalle ab, welche nach dem $\mathrm{Ab}$ waschen und Trocknen bei $235^{\circ}-2400$ schmolzen. Bei einer Prüfung auf Jod konnte ich in einer Probo dasselbe nachweisen. Es schien demnach nur eine Addition von Jodmethyl eingetreten zu sein. Bei einem Versucb, den gebildeten Körper aus heilsem Wasser umzukrystallisieren, schieden sich nach dem Erkalten glänzende Tafeln. vom Schmelzpunkt $235^{\circ}-240^{\circ}$ ab.

Eine von der bei $100^{\circ}$ getrockneten Substanz ausgeführte Elementaraualyse ergab folgende Werte: $0,2357 \mathrm{~g}$ lieferten $0,44 i 2 \mathrm{~g}_{\mathbf{2}}$ und $0,1030 \mathrm{~g} \mathrm{H}_{2} \mathrm{O}$.

Bei einer nach Carius ansgefihrten Jodbestimmung warden aus $0,2639 \mathrm{~g}$ Substanz 0,1322 g Ag $J=27,05$ Proz. J gefunden.

Berechnet für

$\begin{array}{rlr}\mathrm{C}_{19} \mathrm{H}_{19} \mathrm{NO}_{4} \cdot \mathrm{CH}_{3} \mathrm{~J} & \text { Gefunden: } \\ \mathrm{C}=51,39 & 51,71 \\ \mathrm{H} & =4,7 & 4,88 \\ \mathrm{~J} & =27,19 & 27,05\end{array}$

Die gefundonen Werte lehren, dafs auch in alkalischer Lösang nur ein Bulbocapninjodmethyl gebildet war: $\mathrm{C}_{19} \mathrm{H}_{19} \mathrm{NO}_{4} \cdot \mathrm{CH}_{3} \mathrm{~J}$.

Freund und Josephi ${ }^{1}$ ) geben jedoch den Sehmelzpunkt. ihres aus Wasser umkrystallisierten Jodmethylats bei $257^{\circ}$ liegend an; ich habe auch bei den aus Wasser umkrystallisierton glänzenden Tafeln zu wiederholten Malon den Schmelzpunkt nur zwischen $235^{\circ}$ und $240^{\circ}$ gefunden.

Bulbocapnin und Essigsäureanhydrid.

Wenn nach Freund und Josephi im Molekül des Bulbocaprins drei alkoholische Hydroxyle vorhanden sind, so mulste es mit Wahrschoinlichkeit gelingen, drei Acetylgruppen einzuführen.

1) Ann. d. Chem. 277. p. 14. 


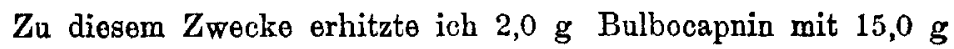
Essigsänreanhydrid und etwa $0,2 \mathrm{~g}$ entwässertem Natriumacetat vier Stunden lang am Steigrohr bis zum schwachen Sieden. Anfangs war die Flüssigkeit ungefärbt, bei weiterer Einwirkung trat jedoch eine prachtvolle, blaue Fluorescenz ein. Nach vierstündigem Erhitzen verjagte ich das Essiggäureanhydrid auf dem Dampfbade, nahm den Rückstand mit Essigäther auf, filtrierte die ätherische Lơsung vom ausgeschiedenen Natriumacetat $a b$ und überliels sie der freiwilligen Verdunstung. Die zurückbleibende weilse Krystallkruste löste ich ohne Erwärmen in absolutem Alkohol, versetzte die schön blau fluorescierende Flüssigkeit mit soviel Wasser, dals sie eben noch klar blieb, und liefs sie in der Kälte verdunsten. Beim Stehen schieden sich kleine, weifse Nadeln $a b$, die sich zu kleinen Büscheln anordneten.

Bei der Elementarmalyse im Kupferoxydrohr fand ich aus $0,2078 \mathrm{~g}$ vorher in Exsiccator gotrockneter Substanz 0,5083 $\mathrm{g} \mathrm{CO}_{2}$ und 0,1037 g $\mathrm{H}_{2} \mathrm{O}$.

$\begin{array}{ccc} & \mathrm{C}_{19} \mathrm{H}_{16} \mathrm{NO}_{4}\left(\mathrm{C}_{2} \mathrm{H}_{3} \mathrm{O}\right)_{3} & \text { Gefunden: } \\ \mathrm{C} & 66,52 & 66,69 \\ \mathrm{H} & 5,54 & 5,53\end{array}$

Nach dem Ergebnis der Elementaranalyse soheint es mir nicht zweifelhaft, dafs $3\left(\mathrm{C}_{2} \mathrm{H}_{3} \mathrm{O}\right)$ in das Bulbocapnin eingetreten sind. Dernnach würde auch die Annahme Freund und Josephi's richtig sein, dafs das Bulbocapnin die Formel:

$$
\mathrm{C}_{18} \mathrm{H}_{13} \mathrm{~N}\left(\mathrm{O} . \mathrm{CH}_{3}\right)(\mathrm{OH})_{3}
$$

hat. Und dem triacetylierten Bulbocapnin würde die Formel

$$
\mathrm{C}_{18} \mathrm{H}_{13} \mathrm{~N}\left(\mathrm{O} . \mathrm{CH}_{3}\right)\left(\mathrm{O} . \mathrm{OC}_{2} \mathrm{H}_{3}\right)_{3}
$$

zukommen.

Salzsaurestriacetyliertes Bulbocapnin.

Versetzt man eine kalte alkoholische Lösung des triacetylierten Bulbocapnins mit Salzsäure bis zur schwach sauren Reaktion und darauf mit Wasser bis zur eben wieder verschwindenden Trübung und überlärst die Flüssigkeit der freiwilligen Verdunstung, so scheiden sich allmälig kleine, weifse, büschelig angeordnete, den Krystallen der freien Base sehr ähnliche Nadeln aus.

Den Chlorgehalt dieser Krystalle versuchte ich auf verschiedeno Weise zu bestimmen. 
I. $0,2091 \mathrm{~g}$ Substanz verloren bei $10000,0316 \mathrm{~g}=15,11$ Proz.

0,1775 der bei $100^{\circ}$ getrockneten Substanz lieferten nach Carius 0,0052 g Ag $\mathrm{Cl}=0,62$ Proz. Cl.

Offenbar war bei dem Trocknen bei $100^{\circ}$ nicht allein Krystallwasser, sondern auch Salzsäure abgespalten. Es ist demnach das triacetylierte Bulbocapnin, wie erwartet werden mulste, eine sehr schwache Base. Berechnet für $\mathrm{C}_{19} \mathrm{H}_{16} \mathrm{NO}_{4}$. $\left(\mathrm{O} \mathrm{C}_{2} \mathrm{H}_{3}\right)_{3}$. $\mathrm{H} \mathrm{Cl}$

$$
\mathrm{Cl}=7,28 \text { Proz. }
$$

$$
\text { Gefunden } \mathrm{Cl}=0,62 \text {, }
$$

II. Eine andere Probe trocknete ich aber Schwefelsäure bis zum konstanten Gewicht. Dabei verloren $0,2555 \mathrm{~g}$ Substanz 0,0298 g $_{3} \mathrm{O}$ $=11,66$ Proz. $\mathrm{H}_{2} \mathrm{O}$.

$0,2257 \mathbf{g}$ derartig getrockneter Substanz ergaben $0,0349 \mathbf{g ~ A g ~ C l}$ $=3,85$ Proz. Cl berechnet für getrocknete Substanz,

$$
=3,36 \text {. Cl " wasserhaltige Substanz. }
$$

Ich führte diesmal die Chlorbestimmung so aus, dals ich das getrocknete Salz in wenig starkem Alkohol löste, zerriebenes Silbernitrat und starke Salpetersäure zufügte, die Mischung mit viel Wasser verdünnte, einmal aufkochte und das ausgeschiedene $\mathrm{Ag}$ Cl abfiltrierte.

III. Zum dritten Male versuchte ich, den Chlorgehalt aus dem lufttrockenen Salz zu bestimmen. Ich verfuhr dabei in der Weise, dals ich eine bestimmte Menge der Substanz direkt in ein Becherglas abwog, dieselbe mit überschüssigem, zerriebenen Silbernitrat mischte, starke Salpetersäure, Wasser und wenig Alkohol zusetzte, die Flüssigkeit aufkochte und das auggeschiedene $\mathrm{Ag} \mathrm{Cl}$ abfiltrierte.

$0,2494 \mathrm{~g}$ luftrockener Substanz lieferten $0,036 \mathrm{~g} \quad \mathrm{Ag} \mathrm{Cl}$ $=3,56$ Proz. Cl.

IV. Zu dieser Analyse verwendete ich ein später, aber anf gleiche Weise dargestelltes Präparat. Die Bestimmung colbst führte ich mit lufttrockenem Material nach Carius aus.

0,2817 g Substanz ergaben $0,0414 \mathrm{~g} \mathrm{Ag} \mathrm{Cl}=3,62$ Proz. Cl.

Die bei Analyse I gofundenen Daten können nicht in Betracht kommen. Hingegen stimmen die bei II, III und IV gefundenen Werte auf lufttrockewes Salz berechnet, annähernd überein:

$$
\begin{array}{rrr}
\multicolumn{3}{c}{\text { Gefunden : }} \\
\mathrm{H}_{2} \mathrm{O}=11,66 & \text { III. } & \text { IV. } \\
\mathrm{Cl}=3,36 & 3,56 & - \\
\hline
\end{array}
$$

Nach diesen Daten aber eine Formel aufzustellen, dürfte gewagt sein, wenn dieselben auch auf die eines basischen Salzes:

$$
2\left[\mathrm{C}_{19} \mathrm{H}_{16} \mathrm{NO}_{4}\left(\mathrm{O} \mathrm{C}_{2} \mathrm{~B}^{3}\right)_{3}\right] \mathrm{H} \mathrm{Cl}+6 \mathrm{H}_{2} \mathrm{O}
$$

einigermafsen stimmen. Ein solches verlangt

$$
\begin{aligned}
\mathrm{H}_{2} \mathrm{O} & =10,32 \text { Proz. } \\
\mathrm{Cl} & =3,39
\end{aligned}
$$


Platinchlorid und triacetyliertes Bulbocapnin.

Säuert man eine in absolutem Alkohol bereitete Lösung des triacetylierten Bulbocapnins mit Salzsäure stark an und fügt Platinchlorid im Ueberschuls zu, so scheiden sich beim Verdunsten kleine, hellgelb gefürbte Nadeln ab. Nach dem Absaugen, Abspülen mit Alkohol und Trocknen zwischen Flielspapier sehen sie wie Nadeln des salzsauren Salzes aus, welche durch Platinchlorid gelb gefärbt sein könnten.

I. $0,2105 \mathrm{~g}$ verloren bei $100^{\circ}$ bis zum konstarten Gewicht getrocknet $0,0105 \mathrm{~g}=4,98$ Proz und hinterlielisen $0,0224 \mathrm{~g}$ Pt $=10,64$ Proz. Pt berechnet auf luftrock $\bullet$ ne Substanz.

Weitere Analysen führte ich aus mit Material späterer Darstellung, welches $1,2,3$ und 4 mal aus mälsig erwärnten, mit Salzsäure und Platinchlorid versetzten Alkohol umkrystallisiert war.

II. $0,2408 \mathrm{~g}$ verloren bei $100^{\circ} 0,0066 \mathrm{~g}=2,74$ Proz. und lieferten $0,0288 \mathrm{~g} \mathrm{Pt}=11,96$ Proz. Pt.

III. $0,2880 \mathrm{~g}$ luftrockenes Salz ergaben $0,0349 \mathrm{~g} \mathrm{Pt}=12,11$ Proz. Pt.

IV. $0,2072 \mathrm{~g}$ lufttrockenes Salz lieferten $0,0267 \mathrm{~g}$ Pt $=12,88$ Proz. Pt.

V. 0,2229 g lufttrocknes Salz hinterlielsen $0,0283 \mathrm{~g} \quad \mathrm{Pt}=$ 12,69 Proz. Pt.

$$
\begin{array}{ccccc}
\multicolumn{7}{c}{\text { Gefunden: }} \\
\mathrm{Pt}=10,64 & \text { II. } & \text { III. } & \text { IV. } & \text { V. } \\
\mathrm{Pt}= & 12,11 & 12,88 & 12,69
\end{array}
$$

Wenn auch durch wiederholtes Umkrystallisieren der Pt-Gehalt des Präparates nicht unwesentlich gesteigert wird, so entspricht or doch nicht dem berechneten. Das Platinsalz:

$$
\left(\mathrm{C}_{19} \mathrm{H}_{18} \mathrm{NO}_{4}\left(\mathrm{OC}_{2} \mathrm{H}_{3}\right)_{3} 2 \mathrm{HCl} \mathrm{Pt} \mathrm{Cl}_{4}\right.
$$

würde 14,82 Proz, enthalien.

$$
\begin{gathered}
\text { Corycavin. } \\
\mathrm{C}_{23} \mathrm{H}_{23} \mathrm{NO}_{6} .
\end{gathered}
$$

Birsmann $n^{1}$ ) scheint, nach don Krystallformen des salzsauren und salpetersauren Salzes seiner $\beta$-Base zu urteilen, bereits Corycavin dargestellt zu haben. Freund und Jos e ph $\mathrm{i}^{2}$ ) haben dasselbe jedoch zuerst in gröfserer Menge und in analysierbarer

1) Inaug. Dissert. Dorpat 1892.

2) Annal. d. Chem. 277 p. 15. 
Form erhalten, ihm auch den Namen Corycavin zuerteilt. Nach ihren Angaben schmilzt dasselbe bei $214^{\circ}-215^{\circ}$. Ich fand jedoch den Schmelzpunkt, sowohl bei dem von $\mathrm{N}$ o ell e stammenden Präparat, als auch bei der von mir selbst dargestellten, aus Alkohol umkrystallisierten Base wiederholt bei $216^{0}-217^{\circ}$.

In Aikohol ist das Corycavin bedeutend schwerer löslich als das Corydalin und krystallisiert daraus in rhombischen, äurserst lichtempfindlichen Tafeln. Aus seinen Salzlösungen wird es durch kohlensaure und Aetzalkalien gefällt; in einem Ueberschusse derselben ist es unlöslich. Freund und $\mathrm{J}_{0} \mathrm{seph}^{1}$ ) sind aufGrund ihrer Elementaranalysen zu der Annahme gelangt, dafs dem Corycavin die Formel $\mathrm{C}_{23} \mathrm{H}_{23} \mathrm{NO}_{5}$ zuzuerteilen sei. Sie geben diese Formel jedoch nur als eine vorläufige bekannt, weil sie aus Mangel an Substanz keine weiteren Analysen ausführen konnten. Sie nehmen jedoch bei allen von ihnen dargestellten Salzen ein Molekül Krystallwasser an, obwohl sie dasgelbe beim Trocknen bei $100^{\circ}$ und selbst bei $110^{\circ}$ vicht nachweisen können. Sie glauben nur auf diese Weise die Zusammensetzung der Salze mit der Formel der freien Base in Einklang bringen zu können.

Nach den Resultaten meiner Elementaranalysen muls ich annehmen, dafs dem Corycavin die Formel $\mathrm{C}_{23} \mathrm{H}_{23} \mathrm{NO}_{6}$ zukommt. Mi dieser Formel wäre auch zugleich der Zusammenhang zwischen del Analysen der Base selbst und der Salze hergestellt.

I. Bei der Elementaranalyse im beiderseits offenen Kupferoxyd rohr mit rorgelegter reduzierter Kupferspirale lieferten $0,275 \mathrm{~g}$ Substany $0,679 \mathrm{~g} \mathrm{CO}_{2}$ und $0,1386 \mathrm{~g} \mathrm{H}_{2} \mathrm{O}$.

II. $0,251 \mathrm{~g}$ Subst. ergaben, in gleicher Weise verbrannt, $0,622 \mathrm{~g}$ $\mathrm{CO}_{2}$ uud $0,1298 \mathrm{~g} \mathrm{H}_{2} \mathrm{O}$.

IlI. $0,2161 \mathrm{~g}$ Subst. ergaben $0,5367 \mathrm{~g} \mathrm{CO}_{2}$ und $0,1109 \mathrm{~g} \mathrm{H}_{2} \mathrm{O}$.

IV. $0,218 \mathrm{~g}$ Subst. in einem mit gemischtem Kupferoxyd und Bleichromat gefüliten Rohre verbrannt lieferten $0,5414 \& \mathrm{CO}_{2}$ und $0,1128 \mathrm{~g} \mathrm{H}_{2} \mathrm{O}$.

V. Bei der N-Bestimmung nach Will und Varrentrapp gebrauchten 0,3012 g Subst. $8,2 \mathrm{~cm} 1 / 10 \mathrm{~N} . \mathrm{HCl}=3,81$ Proz. N.

Berechnet für :

$\begin{array}{cccccc}\mathrm{C}_{23} \mathrm{H}_{23} \mathrm{NO}_{6} & \text { I. } & \text { II. } & \text { III. } & \text { IV. } & \text { V. } \\ \mathrm{C}=67,48 & 67,31 & 67,56 & 67,70 & 67,70 & - \\ \mathrm{H}=5,62 & 5,6 & 5,69 & 5,69 & 5,73 & - \\ \mathrm{N}=3,42 & - & - & - & - & 3,81\end{array}$

1) Annal, d. Chem. 277 p. 16.

Arch. d. Pbarm. CCXXXiv. Bds. 7. Heft. 
Freund und $J$ osephi haben

berechnet

fur $\mathrm{C}_{23} \mathrm{H}_{23} \mathrm{NO}_{5}$

$\mathrm{C}=70,23$

$\mathrm{H}=5,85$ and gefunden :

I.

70,79

6,04
II.

70,46

6,05 .

Salzsaros Corycavin.

$$
\mathrm{C}_{23} \mathrm{H}_{23} \mathrm{NO}_{6} \text {. } \mathrm{HCl} \text {. }
$$

Das salzsaure Salz erhält man durch Auflösen der Base in salzsäurehaltigem heifsen Wasser. Aus der Lösung krystallisiert es in farblosen, derben, zu Rosetten angeordneten Nadeln.

Das Salz erwies sich wasserfrei.

1. Bei der Elementaranalyse im Bleichromatrohr lieferten $0,2083 \mathrm{~g}$ Subst. $0,4722 \mathrm{~g} \mathrm{CO}_{2}$ und $0,1063 \mathrm{~g} \mathrm{H}_{2} \mathrm{O}$.

II. $0,2051 \mathrm{~g}$ ergaben $0,0628 \mathrm{~g} \mathrm{Ag} \mathrm{Cl}=\mathbf{7}, 55 \mathrm{Proz}$. $\mathrm{Cl}$.

Berechnet für:

$\mathrm{C}_{23} \mathrm{H}_{23} \mathrm{NO}_{6} . \mathrm{H} \mathrm{Cl}$

$\mathrm{C}=61.95$

$\mathrm{H}=5,38$

$\mathrm{Cl}=7,97$
Gefunden:

I. II.

61,63

5,65

7.55

Nach Freun 1 und Josephi soll dem salzsauren Corycavin dio Formel $\mathrm{C}_{33} \mathrm{H}_{23} \mathrm{NO}_{5} \cdot \mathrm{H} \mathrm{Cl}+\mathrm{H}_{2} \mathrm{O}$ zukommen. Das Corycavinhydrochlorid verliert jedoch selbst bei $110^{\circ}$ nichts an Gewicht.

$$
\begin{aligned}
& \text { Corycaringlatinchlorid. } \\
& \left(\mathrm{C}_{23} \mathrm{H}_{23} \mathrm{NO}_{6} . \mathrm{H} \mathrm{Cl}\right)_{2} \mathrm{Pt} \mathrm{Cl}_{4}+3 \mathrm{H}_{2} \mathrm{O} \text {. }
\end{aligned}
$$

Fügt man zu einer stark salzsauren Lösung der Base Platinchlorid, so fällt ein hellgelber, amorpher Niederschlag aus. Derselbe kann in heifsem Wasser gelöst werden, und scheidet sich dann aus dieser Flüssigkeit das Platinsalz in der Kälte in kleinen, körnigen Krystallen ab.

$0,277 \mathrm{~g}$ verloren bei $10500,0138 \mathrm{~g} \mathrm{H}_{\mathbf{2}} \mathrm{O}$.

$0,2632 \mathrm{~g}$ getrocknetes Salz hinterliefsen $0,0424 \mathrm{~g} \mathrm{Pt}$.

Berechnet für $\left(\mathrm{C}_{23} \mathrm{H}_{23} \mathrm{NO}_{6} \text {. } \mathrm{H} \mathrm{Cl}\right)_{2} \mathrm{PtCl}_{4}+3 \mathrm{H}_{2} \mathrm{O}: \mathrm{H}_{3} \mathrm{O}=4,22$ Proz.

Gofunden $\mathrm{H}_{2} \mathrm{O}=4,98$ Proz.

Berechnot für $\left(\mathrm{C}_{23} \mathrm{H}_{23} \mathrm{NO}_{8} . \mathrm{H} \mathrm{Cl}_{2} \mathrm{Pt} \mathrm{Cl}_{4}: \mathrm{Pt}=16,12\right.$ Proz.

Gefunden $\mathrm{Pt}=16,10$ Proz.

Obwohl Freund und Josephi bei $140^{\circ}$ ebentalls nur 4,38 Proz. Wasser gefunden haben, nehmen sie doch 5 Molekülo Krystallwasser an. 
Verhalteu des Corycaving gegen Jod.

Diesen Versuch stellte ich in ganz analoger Weise wio beim Corydalin und Bulbocapnin an. Ich löste $0,3419 \mathrm{~g}$ Corycavin in $.50 \mathrm{ccm}$ Alkohol und erwärnite die Lösung mit $1,8533 \mathrm{~g}$ getrockneten Jods drei Stunden lang in einer Druckflasche im Dampfbade. Nach der Einwirkung sah die Lösung braun aus, und am Boden hafteten braune Massen. Nach Zusatz von Natriumbicarbonat, Jodkalium und $150 \mathrm{~cm} \mathrm{1/10} \mathrm{N.} \mathrm{Na}_{2} \mathrm{~S}_{2} \mathrm{O}_{3}$-Lösung wurde die Mischung mehrere Tage gelinde erwärmt. Die Flïssigkeit färbte sich auch gelb und die braunen Partikel verschwanden, aber am Boden des Gefälses setzte sich ein grau-weifser Niederschlag ab. Die Mischung wurde zo $500 \mathrm{ccm}$ aufgefüllt, und es wurden alsdann $j e 100 \mathrm{ccm}$ davon zur Titration verwendet, und zwar brauchten sie $26,5 \mathrm{ccm} 1 / 10$ N.J, $500 \mathrm{ccm}$ also $132,5 \mathrm{ccm} \mathrm{1/10} \mathrm{N}$.J. Demnach wäre beinahe kein Jod in Reaktion getreten, jedenfalls keine Menge, die irgend welcher Gleichung entspräche.

Ich konnte auch nachweisen, dafs unverändertes Corycavin in der Flüssigkeit vorbanden war, indem ich den grau-weifsen Niederschlag in salzsäurehaltigem Wasser löste und die Lösung mit Natriumcarbonat übersättigte, Das ausfallende Pulver krystallisiorte ich aus Alkohol um: es schossen aus der Lösung Krystallindividuen sn, welche die Form und den Schmelzpunkt $216^{0}-217^{0}$ des Corycavins hatten.

Es scheint also auch auf das Corycavin das Jod keine Einwirkung zu haben. Es würde daher nach meinen vorläufigen Untersuchungen in keiner näheren Beziebung zum Corydalin und Berberin stehen.

$$
\begin{gathered}
\text { Corybulbin. } \\
\mathrm{C}_{21} \mathrm{E}_{25} \mathrm{NO}_{4} \text {. }
\end{gathered}
$$

Die Angaben, welche $\mathrm{Freund}$ und $\mathrm{J}$ ose $\mathrm{phi}^{1}$ ) iber diese such von ihnen dargestellte Base machen, sind noch sebr ungenau. Sie geben an, dals sie aus einem Corydalin, bezogen aus der

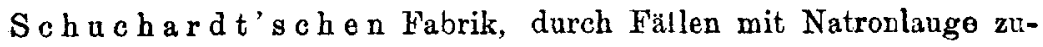
nächst das reine Corydalin entfernt, darauf in die alkalische

1) Ann. d. Chem. 277. p. 18. 
F lü $\mathrm{s}_{\mathrm{s}} \mathrm{gk} \theta \mathrm{it} \mathrm{CO}_{2}$ bis zur Sättigung eingeleitet haben, wodurch eine weitere Fällung entstanden ist. Dieser Niederschlag erwies sich in Alkohol sehr schwer löslich, krystallisierte daraus in filzigen, weirsen Säulen und zeigte den Schmelzpunkt 2070_2080.

Wie ich oben angegeben habe, gelang es mir, aus dem vón Noelle stammenden, zwischen $120^{\circ}$ und $180^{\circ}$ schmelzenden Alkaloidgemisch aut die gleiche Weise eire kleine Mengo einer Base zu isolieren, welche nach dem Unkrystallisicren aus Alkohol ein feinkrystallinisches Pulver darstellte, jedoch bei $235^{\circ}$ schmolz. Ferner habe ich das Extrakt der Corydalisknollen, nach dem Ausschütteln mit Aether, zunächst mit Salzsäure angesäuert, mit Natriumcarbonat alkalisch gemacht, mit Chloroform ausgeschüttelt und daraus gleichfalls Corybulbin vom Schmelzpunkt 238 _-2390. erhalten.

In jüngster Zoit, als ich gerade diese neue Base dargestellt. batte, veröfentlichten $\mathrm{Dob}$ bie und $I$ a $u d e \mathrm{r}^{1}$ ) ihre neuesten Arbeiten über die Corydalisalkaloide. Sie teilen mit, dafs sie aus käuflichem Corydalin-Schuchardt zunächst das Corydalin durch Natriumcarbonat gefällt, darauf in die Lange $\mathrm{CO}_{2}$ im Ueberschufs eingeleitet und den Niederschlag aus Alkohol umkrystallisiert haben. Eine zweite Darstellungsweise aus dom gleichen käuflichen Corydalin ist nach ihren Angaben dio folgende: man entfernt aus dem käutlichen Corydalin das reine Corydalin dureh Ausziehen mit wenig heifsem Alkohol und behandelt den verbleibenden Rückstand mit grofsen Mengen siedenden Alkohols. Aus diesen letzten Lösungen krystallisiert das Corybulbin als fein-krystallinisches Pulver, welches durch Krystallisierenlassen seines Chlorbydrates und Zerlegen desselben mit Ammoniak zu reinigen ist.

Die Angaben stimmen allo darin uberein, dacs dieses neue Alkaloid durch saure kohlensaure Alkalien vollständig gefällt wird und in Alkohol sebr schwer löslich ist. Es wird aber ferner auch gefällt durch Aetzalkalien und durch kohlensaure Alkalien, welcho Fällungsmittel jedoch im starken Ueberschuls angewendet lösend wirken. Leicht löslich ist es ferner in Chloroform, kann aber aus dieser Lösung in schönen weifsen Nadeln erbalten werden. Das

i) Chens. Ztg. 1894. 
Corybulbin ist sehr lichtempfudlich und wird auch schon im Trockenschrank bei $100^{\circ}$ gelb. Der Zersetzungspunkt liegt nach Fre und und Josephi zwischen $207^{\circ}$ and $208^{\circ}$, während Dobbie und $\mathrm{L}$ a u d e $\mathrm{r}$ angeben, dafs es bei $210^{\circ}$ weich wird, aber bei $238^{\circ}$ bis $240^{\circ}$ noch nicht vollständig schmilzt. Nach meinen Beohachtungen schmilzt das aus Chloroform krystallisierte Alkaloid genau zwischen $233^{\circ}$ und $239^{\circ}$ vollstindig. Als Formel baben die englischen Forseher aufgesteilt $\mathrm{C}_{22} \mathrm{H}_{25} \mathrm{NO}_{4}$, wälirend $\mathrm{Fr}$ e und und $\mathrm{J}$ o se phi nur ihre Analysendaten veröffeutlichen, ohne daraus Schlüsse zn ziehen.

$0,2061 \mathrm{~g}$ der im Exsiccator getrockneten Base ergaben bei der Elementaranalyse im Kupferoxydrohre $0,5345 \mathrm{~g} \mathrm{CO}_{2}$ und $0,1332 \mathrm{~g} \mathrm{H}_{2} \mathrm{O}$.
Berechnet für
Gefunden von Freund
Ziegenbein
$\mathrm{C}_{21} \mathrm{H}_{25} \mathrm{NO}_{4}$
$\mathrm{C}=70,98$
$\mathrm{H}=7,04$
und Josephi$$
\text { I. }
$$
71,07
7,2
70.14
7,2
70,72

Sowohl die von Freund und Josephi, als anch die von mir gefundenen Werte sprechen für die Richtigkeit der von den englischen Forschern aufgestellten Formel: $\mathrm{C}_{21} \mathrm{H}_{25} \mathrm{NO}_{4}$. Aus Mangel an Material habe ich nur das salzsaure Salz und das Platindoppelsalz dargestellt, um den Rest mit Jod zu behandeln.

$$
\begin{gathered}
\text { Salzsaures Corybulbin. } \\
\mathrm{C}_{41} \mathrm{H}_{25} \mathrm{NO}_{4} \text {. HCl. }
\end{gathered}
$$

Da das aus den Corydalisknollen dargestellte salzsaure Corybilbin, auch nach häufigem Umkrystallisieren, immer noch eine graue Farbe zeigte, erhitzte ich einen Teil in wässriger Lösung mit wenig rrisch ausgeglühter Tierkohle. Aus dem schwach gelb gefärbten heifsen Filtrat krystallisierten gelbliche, prismatische Nadeln. Dieseyben sind auch in heifsem Wasser schwer löslich, leichter bei Ge renwart von freier Salzsäure.

$0,1479 \mathrm{~g}$ bei $100^{0}$ getrocknet verloren nichts an Gewicht und eranith $0,0541 \mathrm{~g} \mathrm{Ag} \mathrm{Cl}$.

$$
\begin{aligned}
& \text { Berechet fur } \mathrm{C}_{21} \mathrm{H}_{25} \mathrm{NO}_{4}, \mathrm{H} \mathrm{Cl} \\
& \mathrm{Cl}=9,66 \text { Proz. } \\
& \text { Gefunden } \mathrm{Cl}=9,00 \\
& \text { Corybulbinplatinchlorid. } \\
& \left(\mathrm{C}_{21} \mathrm{H}_{25} \mathrm{NO}_{4} \cdot \mathrm{H} \mathrm{Cl}\right)_{2} \mathrm{Pt} \mathrm{Cl}+3 \mathrm{H}_{2} \mathrm{O} \text {. }
\end{aligned}
$$

Beim Versetzen einer möglichst konzentrierten, stark salzsauren Lösung des salzsauren Corybulbins mit überschüssigem Platinchlorid 
fiel ein gelbweifser, amorpher Niederschlag aus, der durch Absauger von der Flüssigkeit getrennt, mit wenig Wasser nachgewaschen und zwischen Fliefspapier getrocknet wurde. Aus der Mutterlange schieden sich nach langem Stehen einige rötlich gefärbte, in der Form dem salzsauren Salz gleichende Krystalle aus.

$0,2396 \mathrm{~g}$ verloren bei $100-105^{0}$ getrocknet $0,0117 \mathrm{~g}$. $0,2279 \mathrm{~g}$ der getrockneten Substanz hinterlielsen $0,0388 \mathrm{~g} \mathrm{Pt}$.

Berechnet für $\left(\mathrm{C}_{21} \mathrm{H}_{25} \mathrm{NO}_{4} . \mathrm{H} \mathrm{Cl}_{2} \mathrm{Pt} \mathrm{Cl}_{4}: \mathrm{Pt}=17,37\right.$ Proz.

Gefunden Pt $=17,02$ Proz.

Berechnet für $\left(\mathrm{C}_{21} \mathrm{H}_{25} \mathrm{NO}_{4} \text {. } \mathrm{H} \mathrm{Cl}\right)_{2} \mathrm{Pt} \mathrm{Cl}_{4}+3 \mathrm{H}_{2} \mathrm{O}: \mathrm{H}_{2} \mathrm{O}=464$ Proz Gefunden $\mathrm{H}_{2} \mathrm{O}=4,88$ Proz.

Nach der gefundenen Wassermenge ist anzunehmen, dafs dem Platinsalz drei Moleküle Krystallwasser zukommen.

Einwirkung von Jod a u Corybulbin.

Schon der Versuch in der Eälte zeigte, dafs Jod auf das Corybulbin reagiert, in ähnlicher Weise, wie es beim Corydalin beobachtet war.

Darauf löste ich 0,2026 getrockneten Corybulbins in circa $60 \mathrm{ccm}$. 96 proz. Alkohol, fügte $1,2720 \mathrm{~g}$ trocknes Jod hinzu und erwärmte in einer Druckflasche drei Stunden lang im Wasserbad.

Nach dem Erkaiten war die Flüssigkeit braun gefärbt und am Boden der Flasche lagen braune, zusarnmengeflossene Massen, die gebildeten Perjodide. Ich fügte unn $90 \mathrm{ccm} 1 / 10 \mathrm{~N}$. $\mathrm{Na}_{2} \mathrm{~S}_{2} \mathrm{O}_{3}$ Liösung $2 \mathrm{~g}$ K J und wenig Natriumbicarbonat zu und erwärmte die Mischung suf dem Dampfbade. Nach wenigen Tagen waren die Perjodile zerlegt, und es resultierte eine intensi gelb gefürbte Flüssigkeit, in der jedoch keine gelb gefärbten Krystalle suspendiert waren. Die gan\%e Flüssigkeit brachte ich in einen $500 \mathrm{ccm}-K o l b e n$ und füllte zur Marke auf. Je $100 \mathrm{ccm}$ der Flüssigkeit titrierte ich mit $1 / 10 \mathrm{~N}$. J-Lösung

Der Umschlag war schwer zu erkernen, zeigte sich nur dureh eine grtunliche Färbung an. Duroh zweimalige Titration stellte ich fest, dafs je $100 \mathrm{~cm} 2,8 \mathrm{ccm} 1 / 10$ N. J-Lös. zur Rücktitration des $\mathrm{Na}_{2} \mathrm{~S}_{2} \mathrm{O}_{8}$ brauchten, $500 \mathrm{ccm}$ also $14 \mathrm{ccm}^{1 / 1}{ }_{10}$ N. J-Lös. Somit waren $90-14=76 \mathrm{ccm}$ $1 / 10 \mathrm{~N}$. $\mathrm{Na}_{2} \mathrm{~S}_{2} \mathrm{O}_{3}$ zur Bindung des von vornherein im Ueberschuls zugesetzten Jods nötig gewesen, oder dieser Ueberschuís betrug 0,0127. 7\&. $=0,9652 \mathrm{~J}$.

Waren ferner $1,2720 \mathrm{~g} \mathrm{~J}$ zugefüg $\dagger$, und $0,9652 \mathrm{~g}$ hatten nicht eingewirlit, so waren $\overline{0,3065 \mathrm{~g}} \mathrm{~J}$ mit $0,2026 \mathrm{~g}$ Corybullin in Reaktion

getreten. 
Der Vorgang dürfte demnach durch folgende Gleichung illustrier: werden können:

$$
\begin{aligned}
\mathrm{C}_{2 \mathrm{t}} \mathrm{H}_{25} \mathrm{NO}_{4}+4 \mathrm{~J} & =\mathrm{C}_{21} \mathrm{H}_{21} \mathrm{NO}_{4} \cdot \mathrm{HJ}+3 \mathrm{HJ} \\
355: 508 & =0,2026: \mathrm{x}
\end{aligned}
$$

Nach obiger Gleichung würden $0,2898 \mathrm{~g} J$ auf $0,2026 \mathrm{~g}$ Corybulbin haben einwirken müssen.

Ich versuchte nun, den gebildeten gelben Körper $\mathrm{zu}$ isolieren, indem ich zunächst die zur Titration verwendeten $200 \mathrm{ccm}$ zur Trockne eindampfte, den Rückstand auf ein Filter brachte mit Wasser auswusch, und das Uebrigbleibende erst in verdünntem, dann in starkem Alkohol zu lösen versuchte. Aus beiden Lösungsmitteln krystallisierte aber kein gelber Körper aus. Die iibrig gebliebenen $300 \mathrm{ccm}$ stellte ich an einen sehr kalten Ort bei Seite: nach einigem Stehen schieden sich schöne, citronengelb gefirbte, 3 bis $4 \mathrm{~mm}$ lange Nadeln aus, grölser als die beim Corydalin ge. bildeten. Doch war die Menge zu gering, um sie analysieren zu können.

Ich erhitzte nun noch einmal eine kleine Menge $(0,2-0,3 \mathrm{~g})$ Corybulbin mit Jod im Ueberschuls und behandelte die Flüssigkeit in gleicher Weise. Die nach dem Erwärmen mit $\mathrm{Na}_{2} \mathrm{~S}_{2} \mathrm{O}_{3}$ re. sultierende gelbe Lösung dampfte ich zur Trockne ein, wusch den Rückstand mit Wasser aus und kochte einen Teil des gelben Pulvers in verdünnt-alkoholischer Lösung mit feuchtem Chlorsilber und Salzsäure. Nach dem Filtrieren und Verdunsten der Lösung zeigten sich kleine, gelbe Nadeln, auch gab die Lösung mit Platinchlorid einen gelben und mit Goldchlorid einen rotbraunen Niederschlag. Der Mangel an Material hinderte mich leider, die Einwirkung des Jods weiter zu studieren.

Schon ein Vergleich der Formeln des Corydalius $\mathrm{C}_{22} \mathrm{H}_{87} \mathrm{NO}_{4}$ und der Corybulbins $\mathrm{C}_{21} \mathrm{H}_{25} \mathrm{NO}_{4}$, nach welchem das Corydalin als Methylcorybuibin anzusprechen wäre, woza noch mehr das Vorhandensein von $3\left(\mathrm{O} . \mathrm{CH}_{3}\right)$-Gruppen $\left.{ }^{1}\right)$ im Corybulbin berechtigt, liefs shnen, dafs das Jod auf beide Alkaloide ähnlich einwirkt. Der Versuch hat gezeigt, dafs auch aus dem Corybulbin unter Austritt von $4 \mathrm{H}$ ein gelber, dem Berberin jedenfalls auch nahe verwandter Körper extsteht.

1) Chem. Ztg. 1894. 


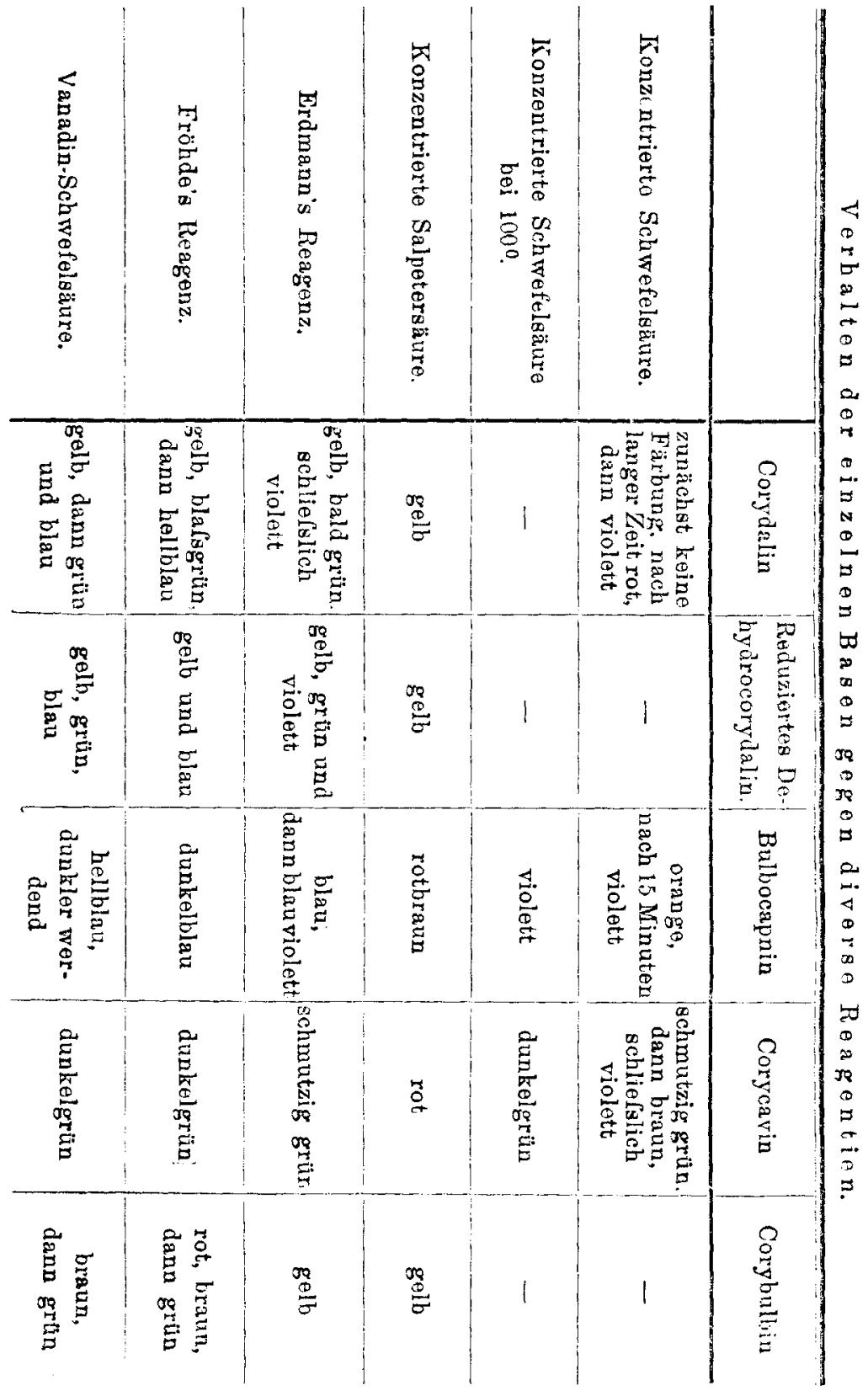

